\title{
Study of the Colocated Dual-Polarized MIMO Capacity Composed of Dipole and Loop Antennas
}

\author{
Dazhi Piao, Xingning Jia, Lingyu Yang, Qingxin Guo, Yi Mao, and Zengrui Li \\ Department of Communication Engineering, Communication University of China, Beijing 100024, China \\ Correspondence should be addressed to Dazhi Piao; piaodazhi@hotmail.com
}

Received 7 January 2015; Revised 12 May 2015; Accepted 17 May 2015

Academic Editor: Angelo Liseno

Copyright (C) 2015 Dazhi Piao et al. This is an open access article distributed under the Creative Commons Attribution License, which permits unrestricted use, distribution, and reproduction in any medium, provided the original work is properly cited.

\begin{abstract}
The colocated dual-polarized dipole (DPD) and dual-polarized loop (DPL) MIMO channel performances are compared. Computation results show that, for the ideal electric and magnetic dipoles, the dual-polarized MIMO systems have identical channel capacity. But the contour plots of the capacity gain of the realistic DPD and DPL are different, due to the difference in antenna patterns. The cumulative distribution function (CDF) of the capacity gain in the two-mirror (TM) channel shows that, for small distance, the capacity gain obtained by the DPD is obviously smaller than that of the DPL, but, with the increase of the distance, the difference gets smaller. A DPL with low mutual coupling is fabricated. Measured results show that high MIMO capacities can be obtained by this DPL in both the anechoic chamber (AC) and the realistic office room. The capacity gain of the DPL antenna is 1.51.99 , which basically coincides with the theoretical and numerical results. Furthermore, the capacity of the virtual DPL antenna with no mutual couplings is also investigated. It is shown that, in the AC, the mutual coupling will generally decrease the dual-polarized MIMO capacity; however, in the office room, the effect of mutual coupling is not always negative.
\end{abstract}

\section{Introduction}

Multipolarized antenna can be used to detect vector electromagnetic field components, which is very attractive to wireless communication, localization, and navigation. The potential of utilizing all the three electric dipoles and three magnetic dipoles for communication which are colocated and orthogonal has been theoretically evaluated in [15]. To obtain high polarization degrees of freedom with the colocated multipolarized antennas, high port isolations between the antennas are generally required. However, it is a challenging task to avoid the mutual coupling between the colocated antennas. Thus, most of the analysis about the colocated multipolarized MIMO system is theory oriented, which is based on the ideal electric and magnetic dipoles with no mutual coupling.

There are also some field test studies using multiple colocated, orthogonal electric dipoles, and it has been verified that multiple independent subchannels could be obtained $[6,7]$. However, due to the constraints of feeding and mutual coupling, the colocated loops are difficult to realize; thus, the measurement based characteristics of the dual-polarized multiple-input multiple-output (MIMO) channel using colocated loop antennas are seldom seen and whether similar MIMO performance can be obtained by the multipolarized loops to that of the multipolarized dipoles has not been verified. Thus, in this paper, firstly, the MIMO capacity of the dual-polarized loop (DPL) is compared with that of the dual-polarized dipole (DPD) by theoretical computations and numerical simulations; then, the colocated DPL antenna is fabricated and its MIMO performance is measured.

A colocated dual-polarized loop antenna has been presented in [8] using a modified classic Kandoian loop, in which the saw tooth interlaced structure is cleverly used to colocate the orthogonal loops. However, this structure introduces a comparatively high coupling between the two loops, and the isolation is about $-13 \mathrm{~dB}$ at the working frequency. In [9], we presented a MIMO antenna composed of two colocated, orthogonal DPLs, which has a constant current distribution along each loop and has a simulated 


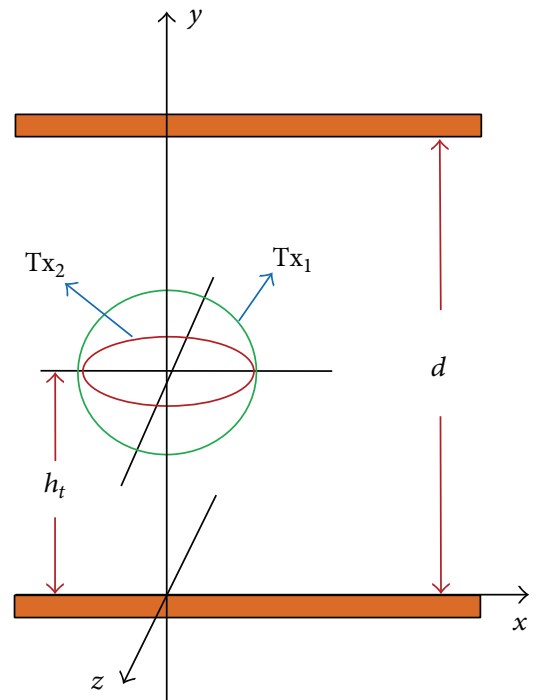

(a)

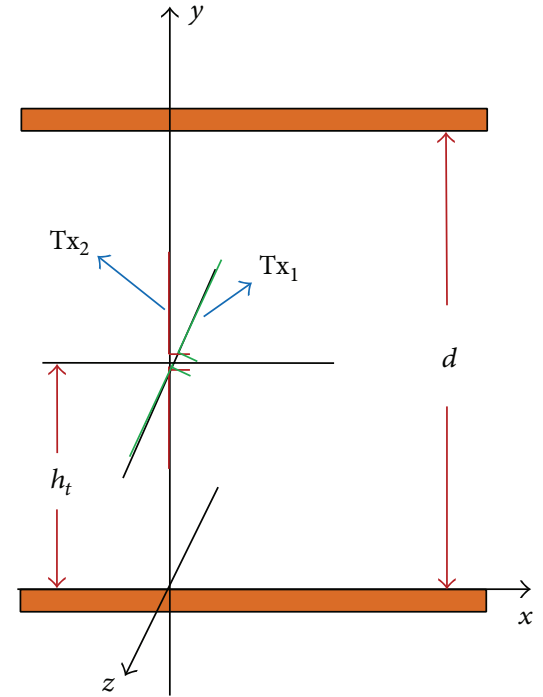

(b)

FIGURE 1: The location of the colocated dual-polarized antenna. (a) The DPL. (b) The DPD.

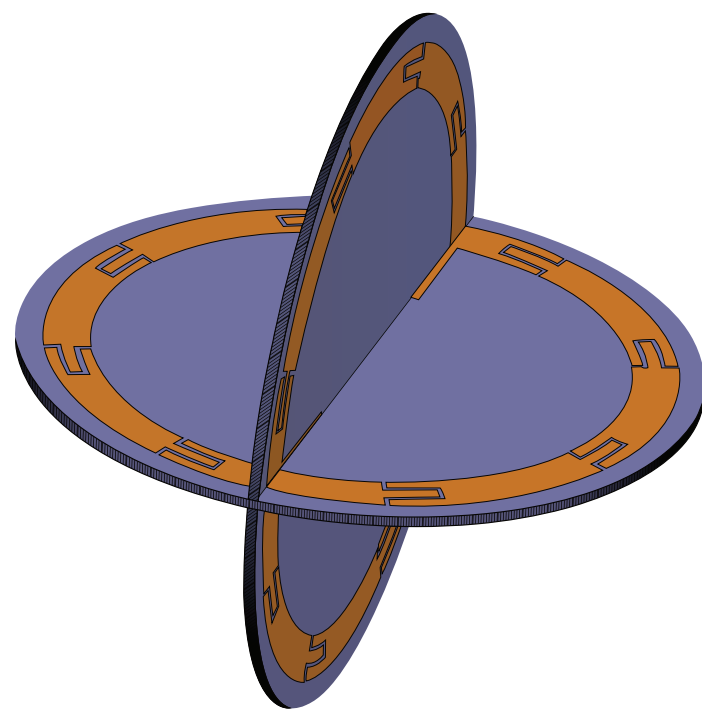

(a)

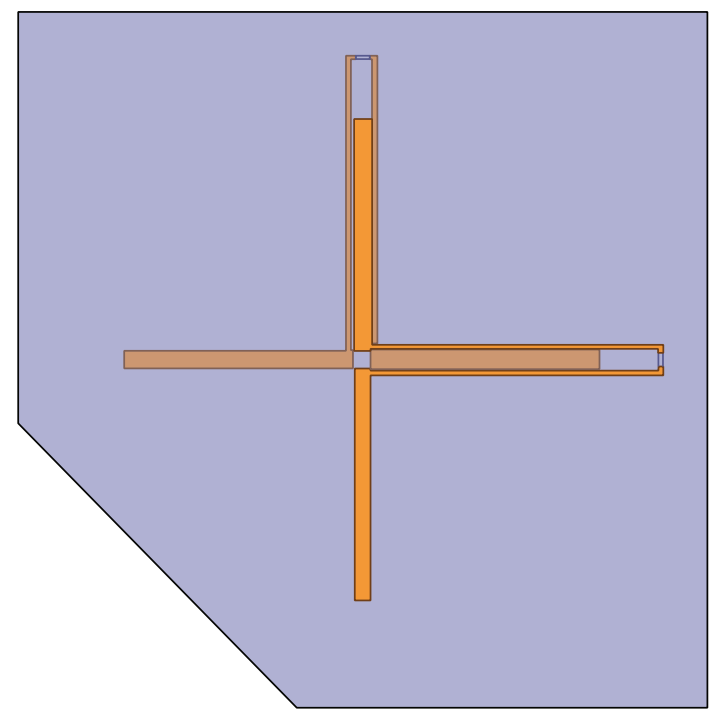

(b)

FIgURE 2: The structure of the colocated dual-polarized antenna. (a) The DPL. (b) The DPD.

mutual coupling lower than $-30 \mathrm{~dB}$. Furthermore, this DPL is compared with another two structures of DPL [10], which have different current distributions and isolations. Simulated results in [10] show that the DPL with constant current distribution and comparatively low mutual coupling has a larger and more robust MIMO capacity. Thus, in this paper, the proposed DPL antenna is fabricated, which has a measured isolation performance better than $-25 \mathrm{~dB}$. Furthermore, in order to study the influence of multipath richness on the information carrying ability of the DPL antenna, the experiments are conducted under an ordinary office environment and an anechoic chamber (AC), individually.
The effects of the communication distance, the transmitting (Tx) and receiving $(\mathrm{Rx})$ antenna heights, and the azimuthal angle between the $\mathrm{Tx}$ and $\mathrm{Rx}$ antennas on the $2 \times 2 \mathrm{MIMO}$ channel characteristics are also evaluated. Furthermore, to study the effect of mutual coupling of the antennas on the dual-polarized MIMO capacity, the measurements based on a virtual DPL antenna are also conducted, which is composed of two single loops having the same polarizations with that of the colocated DPL, but with no mutual couplings. Meanwhile, the MIMO performances of the virtual DPL antennas with other combination of polarizations are also studied, by which the other magnetic components can be utilized. 


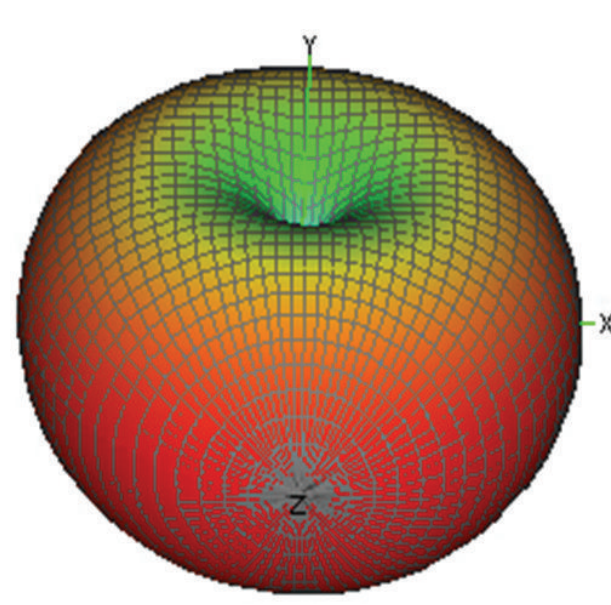

(a)

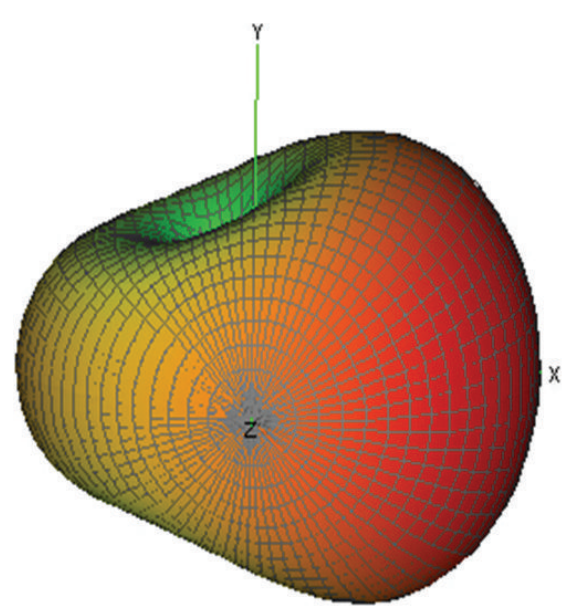

(c)

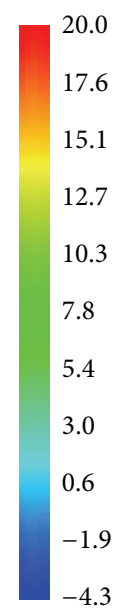

$-4.3$

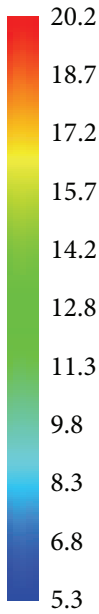

5.3

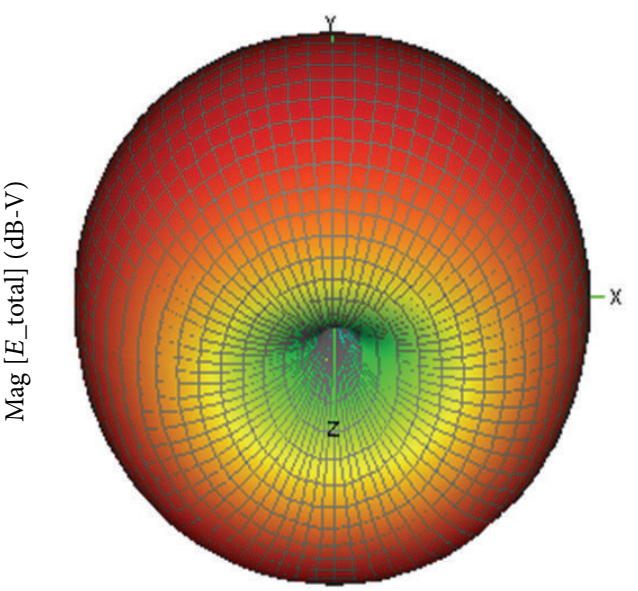

(b)

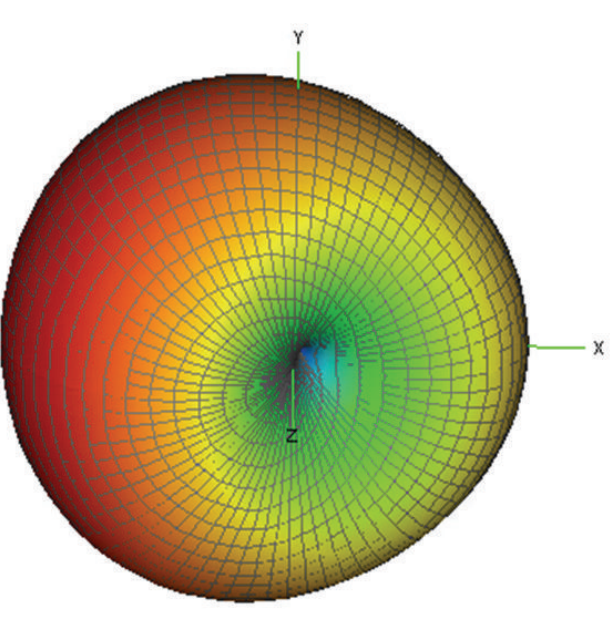

(d)
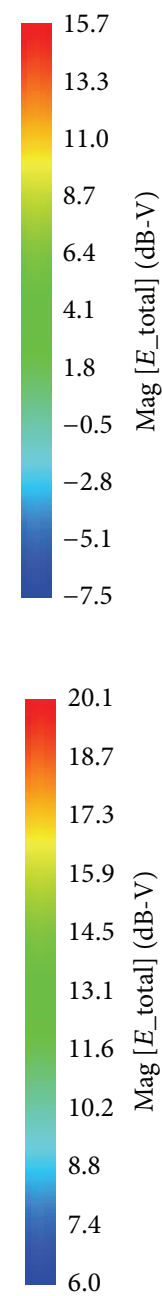

FIGURE 3: The 3D radiation pattern of the antennas. (a) Dipole $y$-polarized. (b) Dipole $z$-polarized. (c) Loop $y$-polarized. (d) Loop $z$-polarized.

\section{MIMO Capacity Comparison between the DPL and the DPD}

The richness of the multipath generally has an important effect on the capacity of a MIMO system. Thus the MIMO performance of the DPL and the DPD antenna is evaluated in free space (FS) and a parallel perfect electric conductor (PEC) two-mirror (TM) channel, respectively. The reason is that, in the FS channel, there is only one multipath, but, in the TM channel, the multipath reflections are infinity; thus two cases of the sparse and rich-multipath environment can be illustrated. Furthermore, for the ideal electric and magnetic dipole, in the FS channel, the exact solution to Maxwell's equation can be found, and, in the parallel PEC TM channel, the electromagnetic (EM) field can be obtained by the image theory [5]. In fact, for the antenna composed of ideal electric and magnetic dipoles, the MIMO system will have similar performance, but, for the antenna composed of realistic electric and magnetic dipoles, the MIMO system may have different behaviours due to the difference in the radiation patterns. Thus, the dual-polarized MIMO channel performance is evaluated by both the theoretical computations considering the ideal electric and magnetic dipoles and the numerical simulations considering the realistic DPD and DPL antenna using the commercial software FEKO.

2.1. Construction of the DPL and DPD Antenna. For the colocated DPL, assume $\mathrm{Tx}_{1}$ is located in the $x y$ plane $(z$ polarized loop) and $\mathrm{Tx}_{2}$ is located in the $x z$ plane $(y$ polarized loop), as shown in Figure 1(a). For the colocated $\mathrm{DPD}$, assume $\mathrm{Tx}_{1}$ is located along the axis $z$ and $\mathrm{Tx}_{2}$ is located along the axis $y$, as shown in Figure $1(\mathrm{~b})$. Here, $h_{t}$ is the distance from the centre of the antenna to the axis $x$. In the TM channel, there are two parallel infinite PEC planes vertical to the axis $y$ with a separation $d$.

As shown in Figure 2, a DPL antenna and a DPD antenna are designed, both of which have a simulated return loss less than $-10 \mathrm{~dB}$ and a mutual coupling lower than $-20 \mathrm{~dB}$ for the working bandwidth centered at $2.4 \mathrm{GHz}$. In order to retain the radiation characteristics of a magnetic dipole, the electric loop should have a constant current distribution. Thus a 


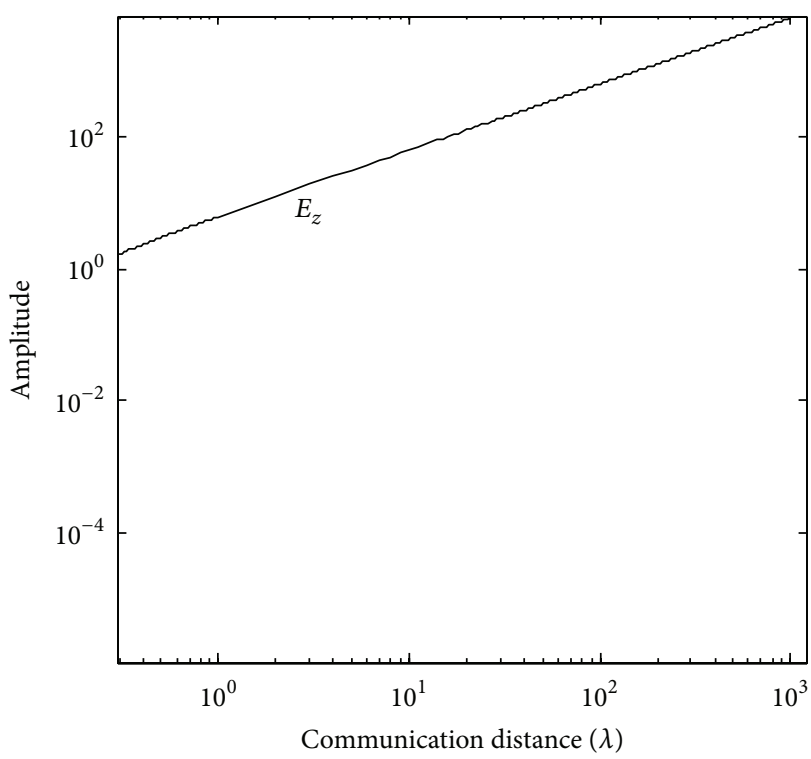

(a)

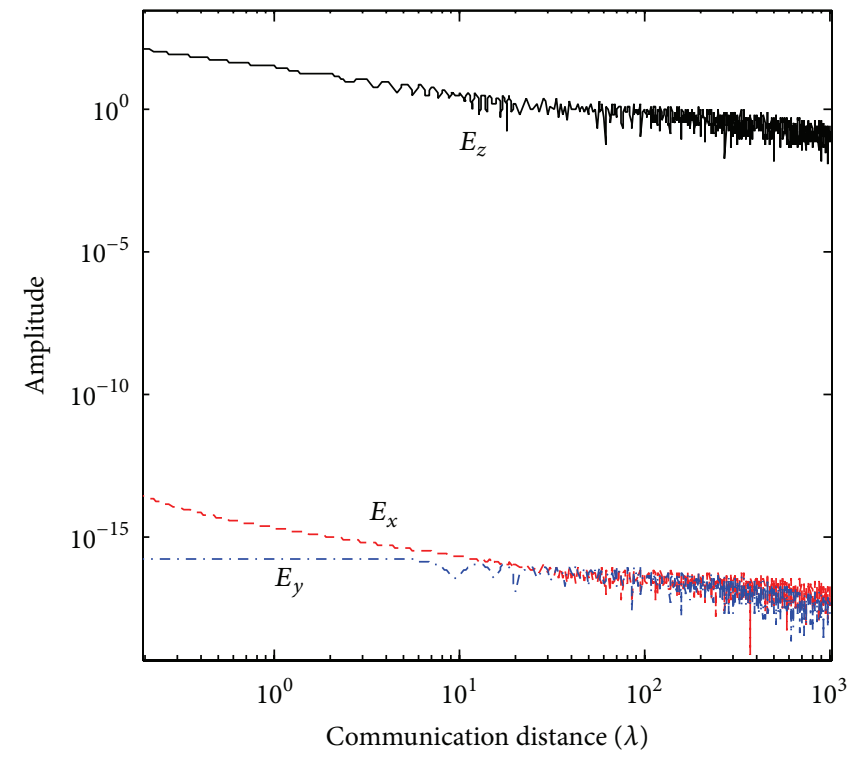

(c)

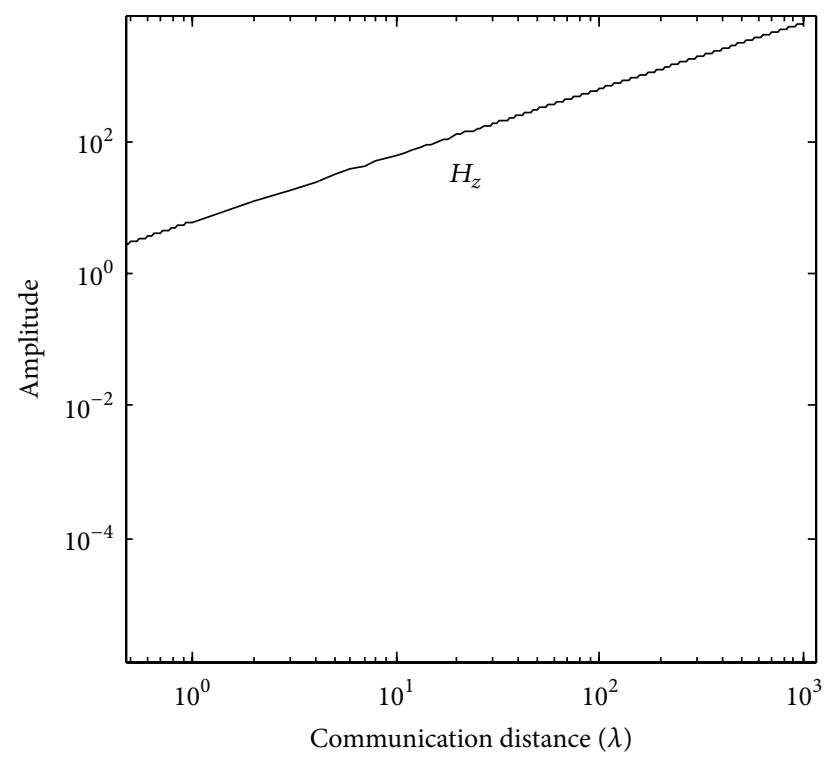

(b)

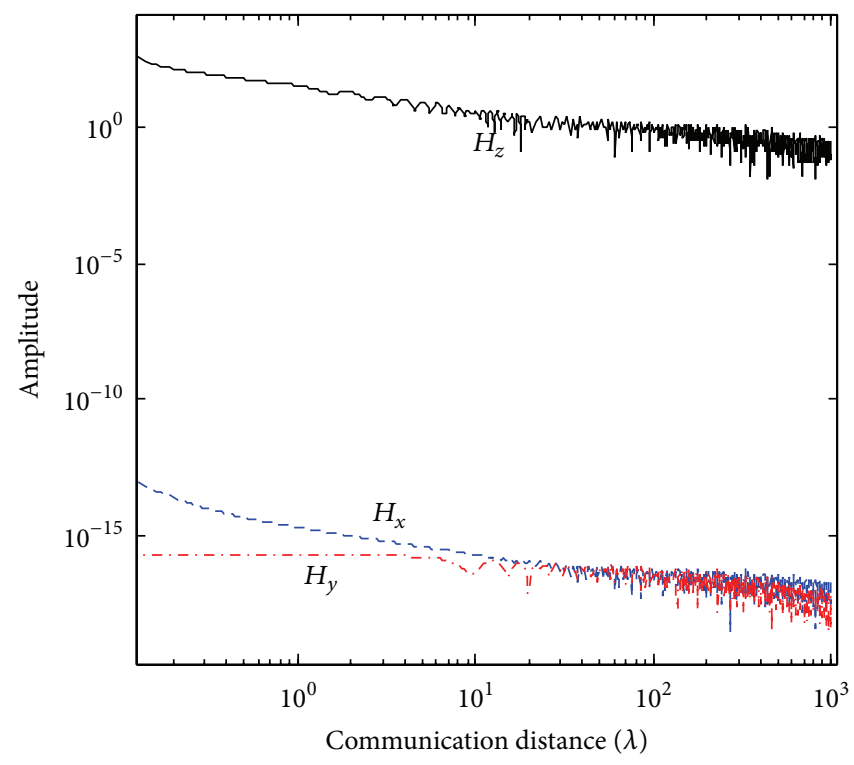

(d)

FIGURE 4: EM field components of the ideal dipole and loop antenna ( $z$-polarized). (a) Dipole in FS. (b) Loop in FS. (c) Dipole in TM. (d) Loop in TM.

structure of loop antenna with a uniform current distribution is selected in this paper [11]. Compared to the Kandoian loop used in the cross-polarized two-loop antenna in [8], the loop here has a better performance in terms of the bandwidth, gain, and radiation efficiency. More importantly, its metal structure all locates near the outer edge of the substrate, and there just exists a gap between the parallel-strip feed lines, as shown in Figure 2(a). Thus, this structure is more convenient to constitute an orthogonal dual-polarized antenna, which has a low mutual coupling.

The basic structure of each single loop is similar to that in [11], which is printed on a Teflon substrate $\left(\varepsilon_{r}=2.6\right.$ and $\tan \delta=0.002)$ with a thickness $h=0.8 \mathrm{~mm}$. It is very important to colocate the loops to ensure that any measured capacity increase in the dual-polarized MIMO system is the result of the polarization effect rather than the spatial diversity. In order to orthogonally colocate the two loops, the design of each single loop is modified by cutting out of a gap between the parallel-strip lines to allow for the insertion of the other loop. Thus the gap width between the parallel-strip lines on the original single loop $g_{p}$ is enlarged from $0.8 \mathrm{~mm}$ to $1.2 \mathrm{~mm}$ to make sure the printed copper strip lines of the two loops do not contact with each other. By the optimization using the full-wave simulation software Ansoft HFSS, the other parameters of each single loop are summarized as follows: outer radius $R_{1}=23.5 \mathrm{~mm}$, inner 


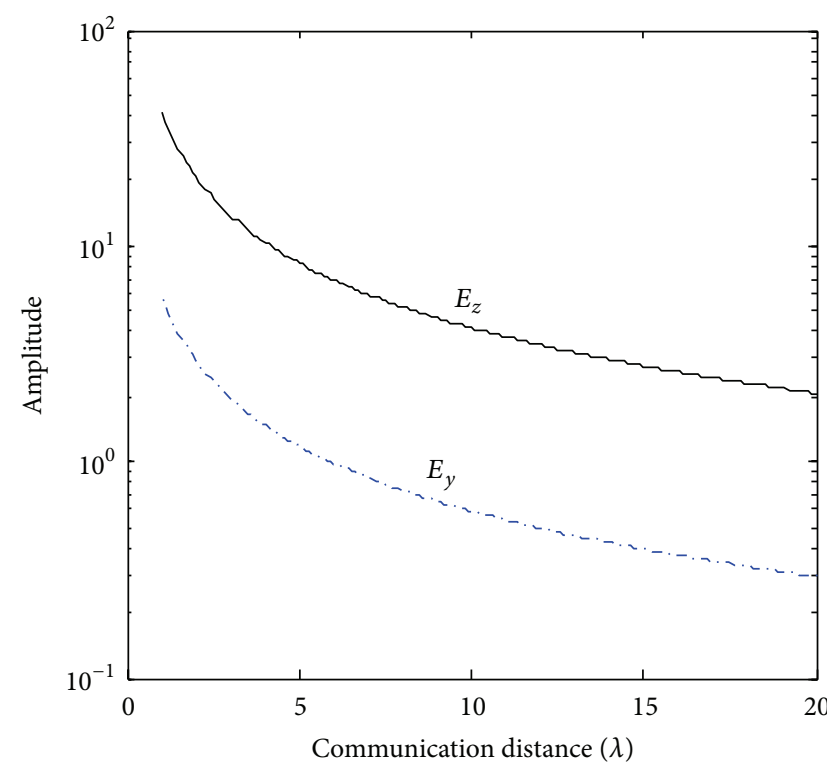

(a)

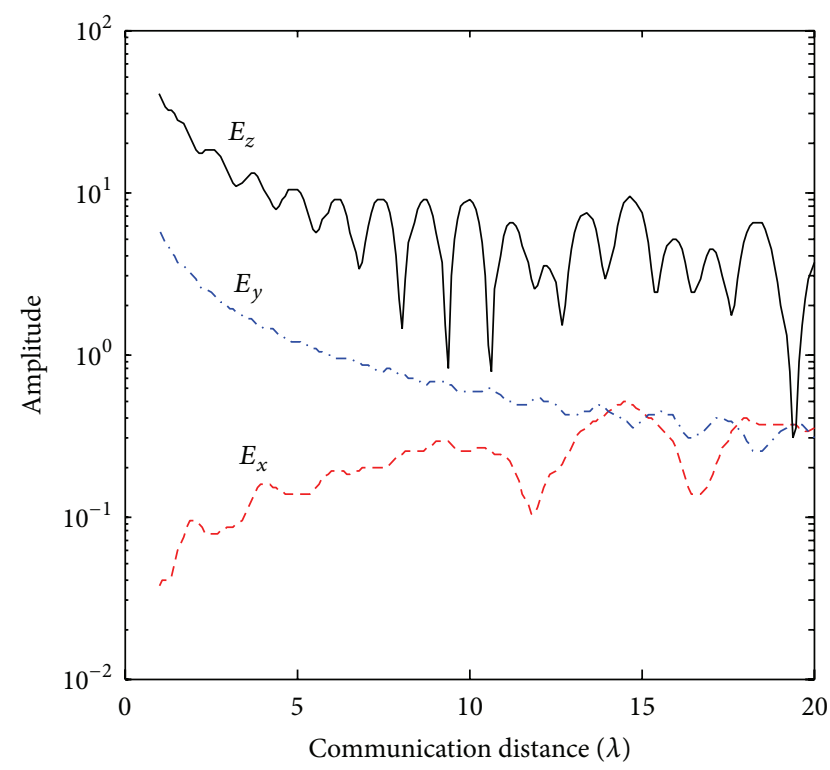

(c)

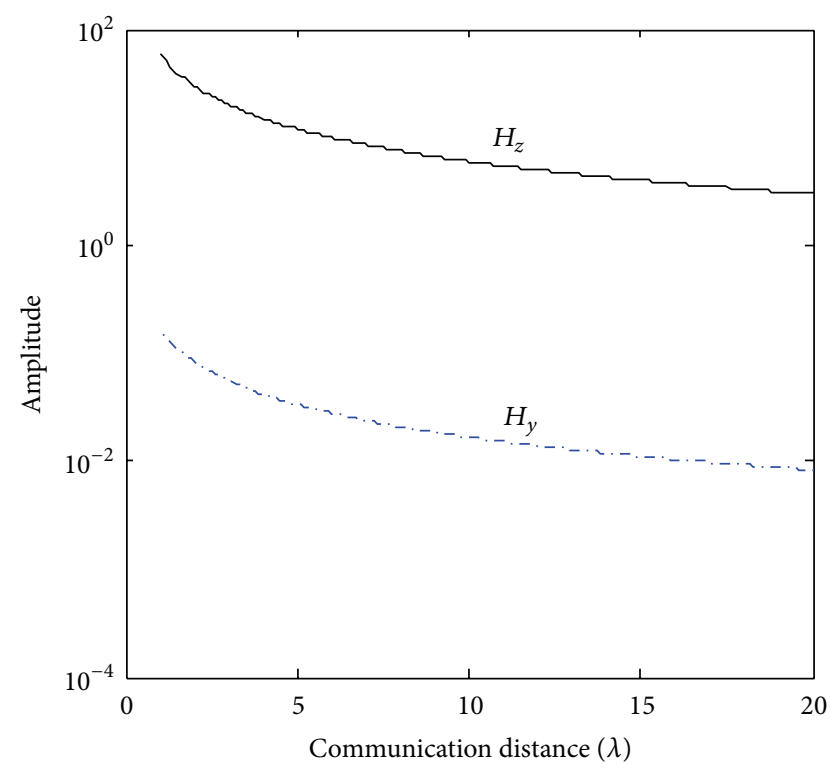

(b)

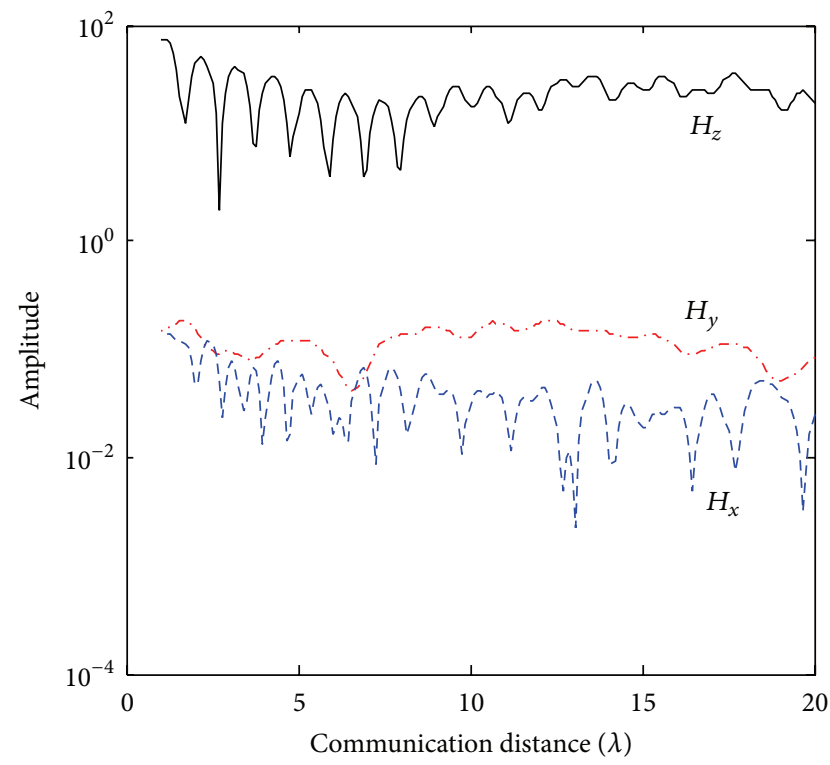

(d)

FIGURE 5: EM field components of the realistic dipole and loop antenna ( $z$-polarized). (a) Dipole in FS. (b) Loop in FS. (c) Dipole in TM. (d) Loop in TM.

radius $R_{2}=20.5 \mathrm{~mm}$, angle of each line section $a_{1}=44^{\circ}$, spacing angle between adjacent line sections $a_{2}=10^{\circ}$, inner bulgy stub width $\omega_{s}=1 \mathrm{~mm}$, separation between the inner bulgy stub and the outer hollow stub $g_{s}=0.4 \mathrm{~mm}$, angle $a_{3}=110^{\circ}$ of the inner bulgy stub, spacing angle $a_{4}=10^{\circ}$, $l_{p}=13 \mathrm{~mm}$, and width $\omega_{p}=0.5 \mathrm{~mm}$. The above parameters have similar meanings to that in [11].

Figure 2(b) shows the configuration of our colocated DPD antenna, which consists of two half-wave dipoles with rectangular arms. They are fabricated orthogonally on each side of the substrate, with the length $L_{a}=55.5 \mathrm{~mm}$, width $W_{a}=2 \mathrm{~mm}$, and both of the two dipoles are fed by a kind of coplanar strip line (CPS), which can reduce the input impedance by enlarging its offset. After optimization, the CPS has the parameters as follows: length $L_{s}=34 \mathrm{~mm}$ and gap $G_{a}=2 \mathrm{~mm}$. The dipoles are printed on a rectangle FR4 substrate with a thickness $h=0.8 \mathrm{~mm}$. The $3 \mathrm{D}$ radiation patterns of the antennas working at $2.4 \mathrm{GHz}$ are shown in Figure 3; we can see that the radiation patterns of the loops have some derivations from that of the ideal magnetic dipole, which may be caused by the effects of mutual couplings.

2.2. Computation and Simulation Results. For each $\mathrm{Tx}$ antenna, there are generally three electric field components 


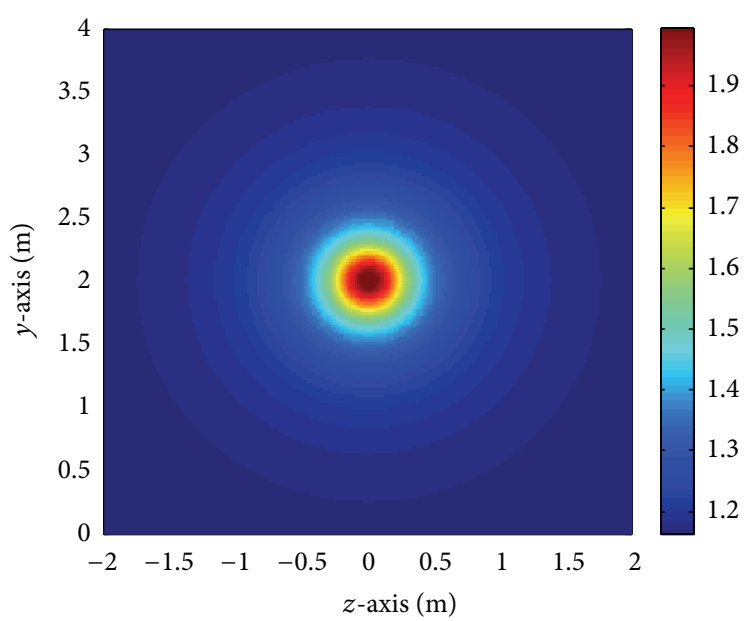

(a)

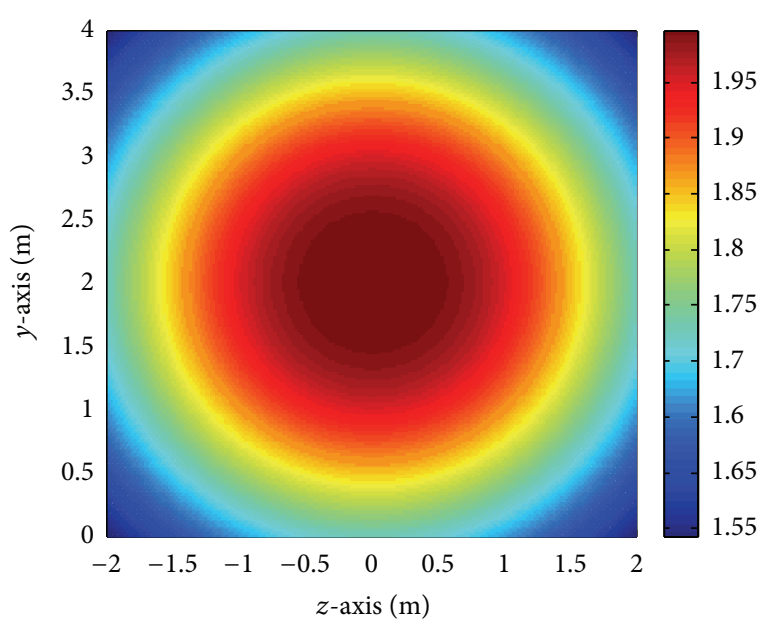

(b)

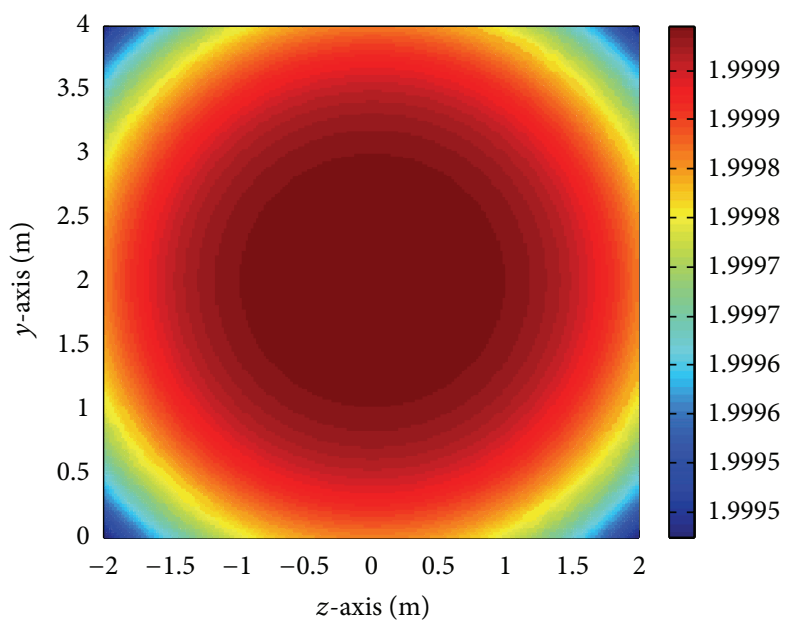

(c)

FIgURE 6: Capacity of the ideal DPD and DPL on the cross section vertical to the $x$-axis in FS. (a) $x=1 \lambda$. (b) $x=10 \lambda$. (c) $x=100 \lambda$.

and three magnetic field components at a Rx point; however, we find that the maximum capacity could be obtained if the field components corresponding to the polarizations of the Tx antennas are employed. In particular, if the dual-polarized channel matrix can be expressed as

$$
\mathbf{H}=\left[\begin{array}{ll}
h_{11} & h_{12} \\
h_{21} & h_{22}
\end{array}\right] \text {, }
$$

considering the dual-polarized antenna configuration shown in Figure 1, for the DPD antenna, $h_{11}$ and $h_{21}$ are the $y$ and $z$ electric field components produced by $\mathrm{Tx}_{1}$, correspondingly, and $h_{12}$ and $h_{22}$ are the $y$ and $z$ electric field components produced by $\mathrm{Tx}_{2}$, correspondingly. For the DPL antenna, the electric field components are replaced by the corresponding magnetic field components. The nonzero electric and magnetic field components are illustrated in Figure 4, produced by the $z$-polarized ideal electric and magnetic dipoles in the FS and the TM channel, respectively. In Figure 5, the field components produced by the realistic dipole and loop antennas are also given. In Figures 4 and $5, h_{t}=2 \mathrm{~m}$, the Tx antennas are fixed, and the Rx points are moving along the axis $x$, with the same height of $2 \mathrm{~m}$. In Figure 5, the communication range is limited to $20 \lambda$, and $\lambda$ is the wavelength in free space at $2.4 \mathrm{GHz}(12.5 \mathrm{~cm})$, due to the limitations of computation capability of the computer. Figures 4 and 5 tell us that, in the TM channel, the number of nonzero field components is increased compared with that in the FS channel.

Channel capacity is defined as the highest achievable bit rate of information that can be sent with arbitrary low probability of error. For the MIMO system with no channel state information (CSI) at the Tx and perfect CSI at the $\mathrm{Rx}$, the equal power transmission strategy is generally employed, whose performance approaches to the optimum power allocation scheme of water-filling. For that case, the capacity of the MIMO system can be computed by [12]

$$
C=\log _{2} \operatorname{det}\left(\mathbf{I}+\left(\frac{\mathrm{SNR}}{n_{T}}\right) \mathbf{H H}^{\dagger}\right),
$$




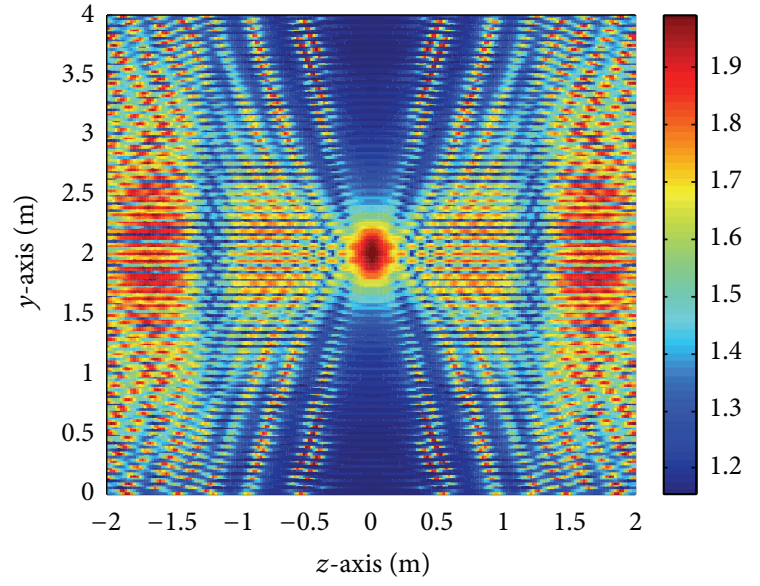

(a)

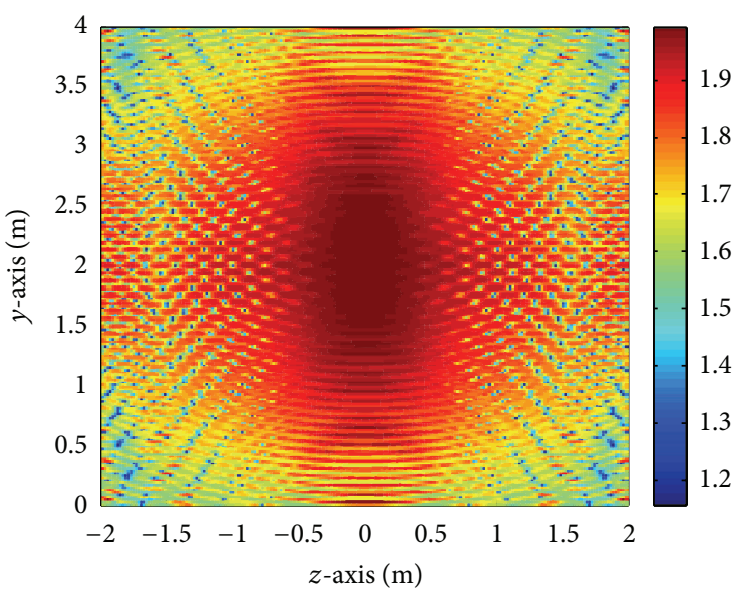

(b)

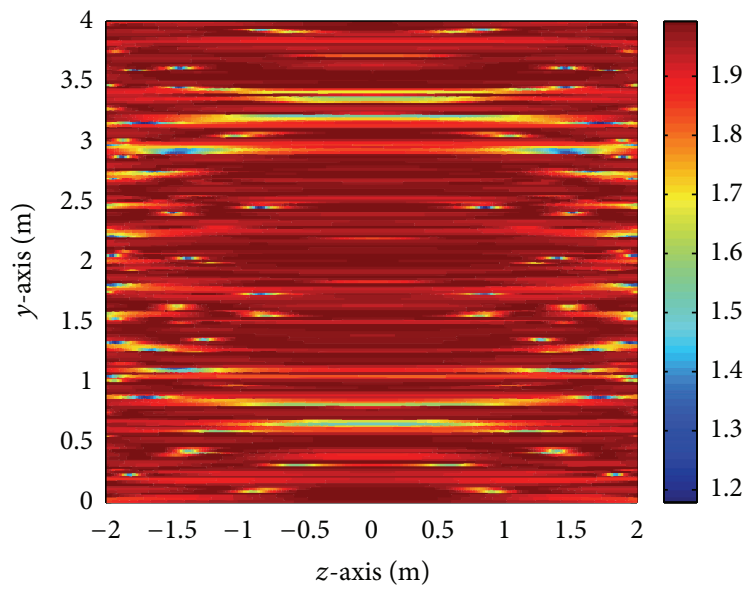

(c)

Figure 7: Capacity of the ideal DPD and DPL on the cross section vertical to the $x$-axis in TM. (a) $x=1 \lambda$. (b) $x=10 \lambda$. (c) $x=100 \lambda$.

where I is a $2 \times 2$ identical matrix, $n_{T}=2$, SNR is the signal to noise ratio (in this paper, we assume a $20 \mathrm{~dB}$ SNR), and $\mathbf{H}^{\dagger}$ means the transpose conjugate of $\mathbf{H}$. We construct a $2 \times$ 2 polarized channel matrix whose element $h_{m n}$ is the channel response measured at the $\mathrm{Rx}$ antenna $m$ generated by the Tx antenna $n$. A widely used channel normalization is adopted, $\|\mathbf{H}\|_{F}^{2}=n_{T} n_{R}$, where $\|\mathbf{H}\|_{F}^{2}$ denotes the Frobenius norm.

The capacity gain of the dual-polarized antenna over onepolarized antenna on the cross section vertical to the axis $x$ is given in Figures 6-11, with different distance to the Tx antenna. As shown in Figures 6 and 7, obtained by theoretical computations, for the ideal electric and magnetic dipoles, the DPD and the DPL have the same capacity results in both the FS channel and the TM channel, because the normalized field components are identical. Furthermore, results in Figure 6 show that, in FS channel, the contour patterns of the capacity gain are regular concentric circles and the largest capacity gain lies in the projection of the point of the Tx antenna on that cross section, which is the centre of the capacity gain contours. On each cross section, with the increase of the distance from the circle centre, the capacity gain gets a monotonous decrease. Furthermore, with the increase of $x$, the radius of the contours of capacity gain gets larger. But in the TM channel, as shown in Figure 7, the contour patterns of the capacity gain are not the regular circles, which have a more complex pattern, due to the interference between the multiple reflections.

In Figures 8 and 9, the capacity gain results obtained by the realistic DPD and DPL antenna in FS are illustrated, individually. It is seen that the contour plots of the DPD and DPL antenna are slightly different, which are not regular concentric circles, and two centres occur in the contour plot. This is probably because the radiation patterns are not the same as that of the ideal electric and magnetic dipoles. In Figures 10 and 11, the capacity gain results obtained by the realistic DPD and DPL antenna in the TM channel are illustrated, individually. It is seen that the contour plots of the DPD and DPL antenna are different; however, both of them are similar to the results in Figure 7, but the contour patterns are more complex in Figures 10 and 11, which is a joint effect of the antenna radiation pattern and the multiple reflections in the channel. Furthermore, the cumulative distribution function (CDF) of the capacity gain of the realistic DPD and DPL antenna is evaluated in Figure 12, with respect to the 


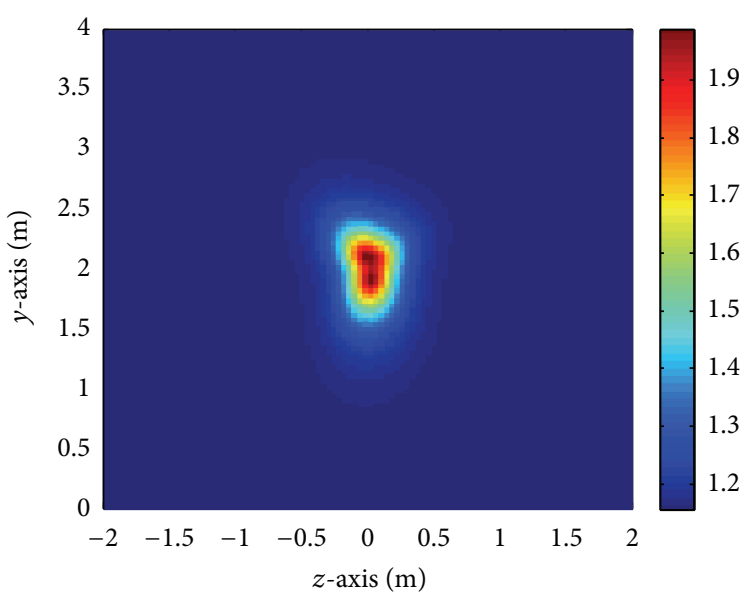

(a)

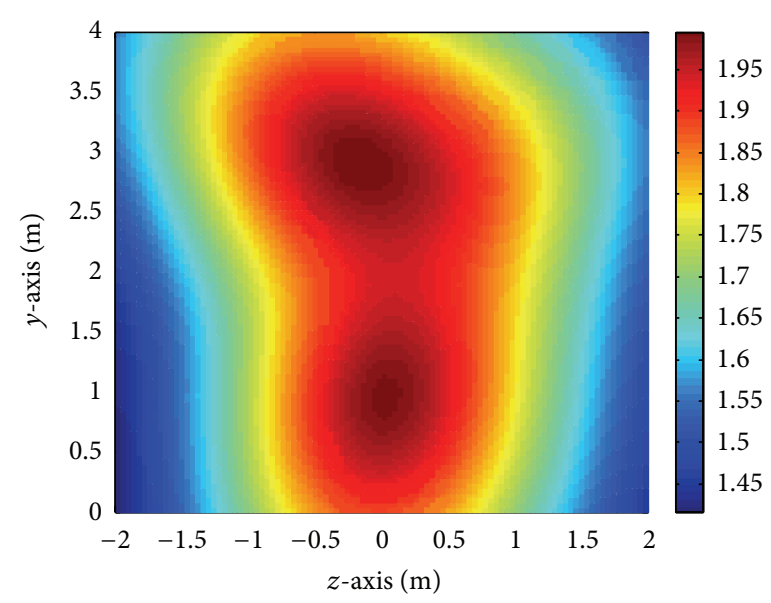

(b)

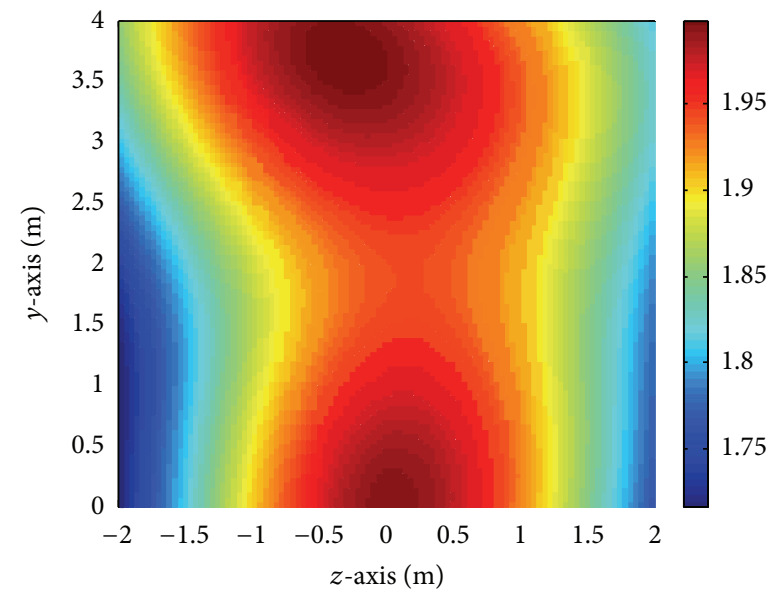

(c)

FIGURE 8: Capacity of the realistic DPD antenna on the cross section vertical to the $x$-axis in FS. (a) $x=1 \lambda$. (b) $x=11 \lambda$. (c) $x=20 \lambda$.

three cross sections in Figures 10 and 11. Figure 12 tells us that, for small communication distance, the capacity gain obtained by the DPD antenna is obviously smaller than that of the DPL antenna, but, with the increase of the communication distance, the difference gets smaller. Specifically, for $x$ larger than $20 \lambda$, the CDFs of the DPD antenna and the DPL antenna almost coincide with each other.

There are already many measurement results corresponding to the DPD antenna; thus only the DPL antenna is fabricated and its MIMO performance is measured in this paper.

\section{Measurement Results of the DPL Antenna}

3.1. Scattering Parameter and Radiation Pattern. The measured scattering parameters and the radiation patterns of the DPL antenna are presented in Figures 13 and 14, respectively. It is shown in Figure 13 that the proposed DPL has a better isolation performance than that in [8]. Specifically, over the working frequency band, the $S_{12}$ and $S_{21}$ in Figure 13 are lower than $-25 \mathrm{~dB}$, whereas the $S_{12}$ and $S_{21}$ are about $-13 \mathrm{~dB}$ in [8]. In Figure 14, the radiation performances of the single loop and the DPL are compared for the working frequency of $2.65 \mathrm{GHz}$. We can see that the radiation patterns of the single loop are very close to that of the magnetic dipole, due to the constant current distribution. The E-planes of the DPL are very close to that of the single loop, but the H-planes of the DPL have some deviations from the omnidirectional one, which might be due to the mutual coupling and feeding. The radiation patterns of the $\mathrm{Rx}$ antenna are similar to that of the Tx antenna; thus, they are not shown here.

3.2. Channel Measurement Experiment Setup. Besides the pattern and the location of the antenna, the capacity of the multipolarized MIMO system depends heavily on the multipath properties of the environment. Different to artificially creating a rich-multipath environment in the laboratory, two typical propagation environments are selected, to test the robustness of the DPL antenna in different application conditions. The first one is an ordinary office room and the second one is an $\mathrm{AC}$, because the $\mathrm{AC}$ can be used to mimic a FS channel, in which there is only one line-of-sight signal. Generally, the multipolarized MIMO system will have a large capacity in the rich-multipath environment and have the 


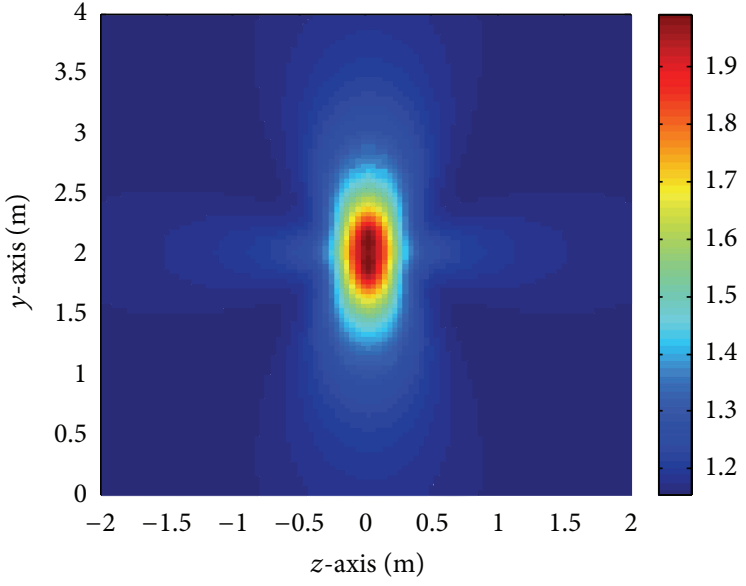

(a)

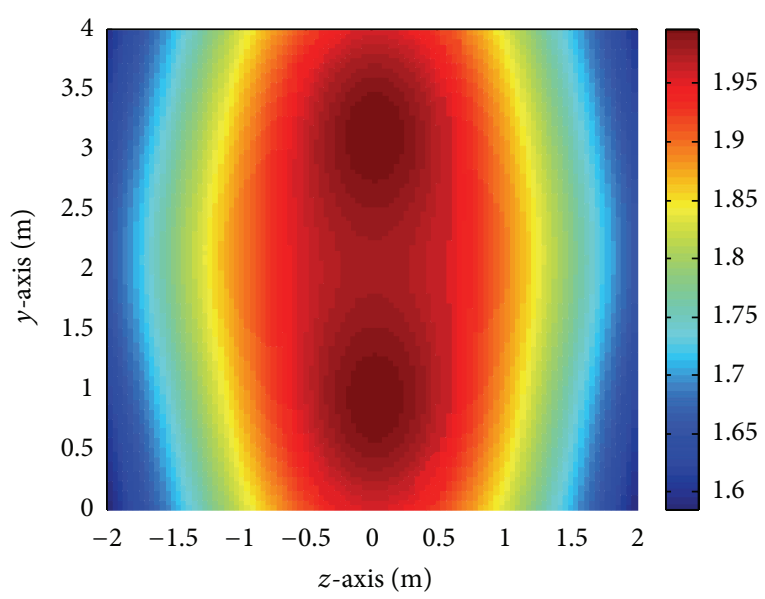

(b)

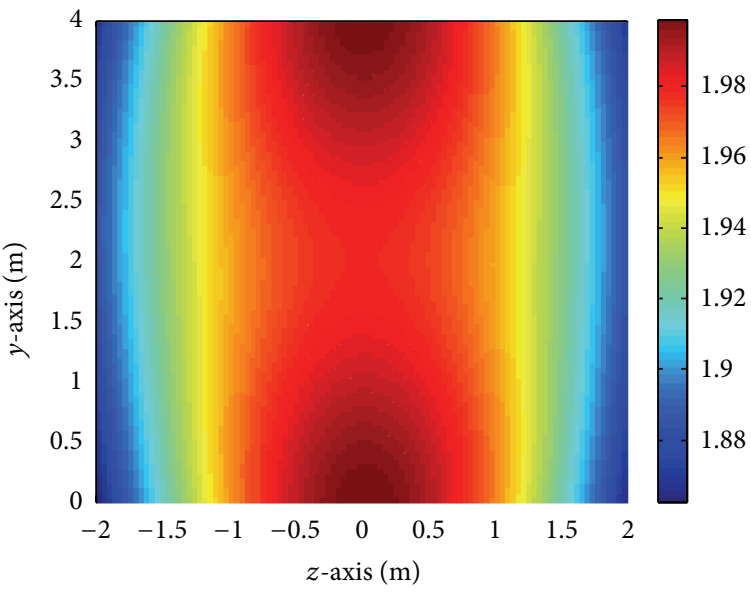

(c)

FIgURE 9: Capacity of the realistic DPL antenna on the cross section vertical to the $x$-axis in FS. (a) $x=1 \lambda$. (b) $x=11 \lambda$. (c) $x=20 \lambda$.

smallest capacity in the FS channel; thus the performance in the AC can be used as a reference for the extremely sparse multipath environment.

A small office room with cement concrete walls is used in the channel measurement, which has rich-multipath propagation. As shown in Figure 15, the room has a length of $2.9 \mathrm{~m}$, a width of $2.7 \mathrm{~m}$, and a height of $3.2 \mathrm{~m}$. The channel path gains are obtained from the $S_{21}$ parameters between the Tx and $\mathrm{Rx}$ antennas. Here, the proposed DPL antenna is used in both the Tx and the Rx ends, and the scattering parameters are obtained by a network analyzer.

During the experiment, for the colocated DPL, $\mathrm{Tx}_{1}$ and $\mathrm{Rx}_{1}$ are located in the $x y$ plane, and $\mathrm{Tx}_{2}$ and $\mathrm{Rx}_{2}$ are located in the $y z$ plane. Here, $h_{t}$ is the Tx antenna height and $h_{r}$ is the $\mathrm{Rx}$ antenna height, $R$ is the horizontal communication distance, $d_{t}$ is the distance between the Tx and the vertical wall having a glass window, and $\zeta$ is the angle between the positive $x$ axis and the distance vector connecting the projections of the Tx and the $\mathrm{Rx}$ on the $x z$ plane. For the virtual DPL, which consists of the combination of two single loops, we firstly put the $\mathrm{Tx}_{1}$ and $\mathrm{Rx}_{1}$ on the $x y$ plane and then put the $\mathrm{Tx}_{2}$ and $\mathrm{Rx}_{2}$ on the $y z$ plane and finally put the $\mathrm{Tx}_{3}$ and $\mathrm{Rx}_{3}$ on the $x z$ plane. Thus, we can get 3 virtual $2 \times 2$ channel matrixes, considering different combinations of the two loops.

\subsection{Capacity Measurement Results of the Colocated DPL} Antenna. In Figure 16, the relationship between the MIMO capacity and the communication distance $R$ is investigated based on the colocated DPL antenna. Here, the Tx antenna is fixed, and the Rx antenna moves along the axis of $x$, and $d_{t}$ is kept as a constant of $1.35 \mathrm{~m}$ in the small room. It is shown in Figure 16(a) that if the Tx and Rx antennas have the same height, the capacity in the realistic office is about $30 \%$ larger than that in AC. However, if the Tx and Rx antennas have different heights, as shown in Figure 16(b), the capacity results in the two scenarios have little difference. With $20 \mathrm{~dB}$ SNR, the single-polarized SISO capacity for the normalized channel matrix is $6.65 \mathrm{bps}$; thus the capacity gains by the DPL antenna are 1.5-1.99, which is basically consistent with the theoretical and the simulated results.

Figure 17 shows that, in both the AC and the small office room, the DPL MIMO channel can obtain two nonzero eigenvalues. Furthermore, the condition number $(\mathrm{CN})$ can also be obtained, which is defined as the ratio between the 


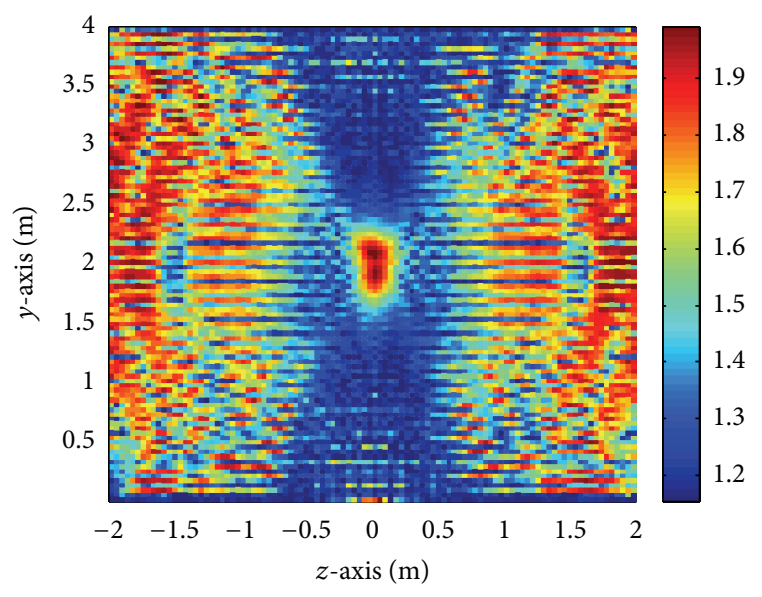

(a)

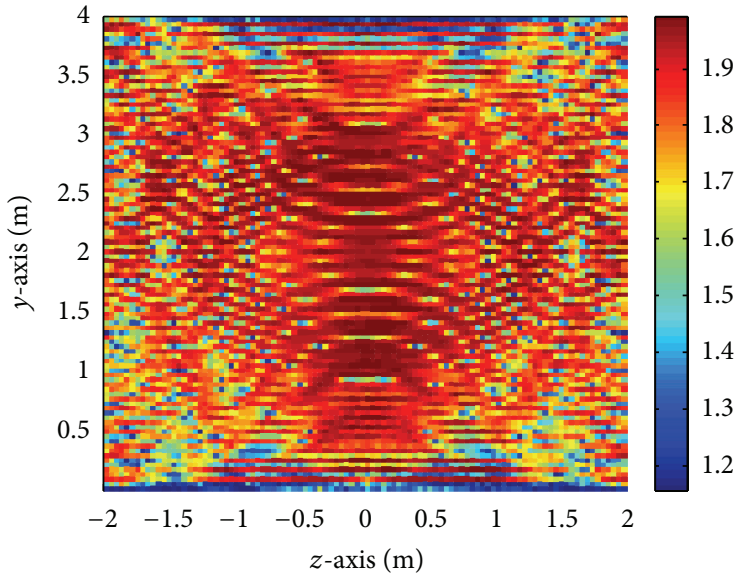

(b)

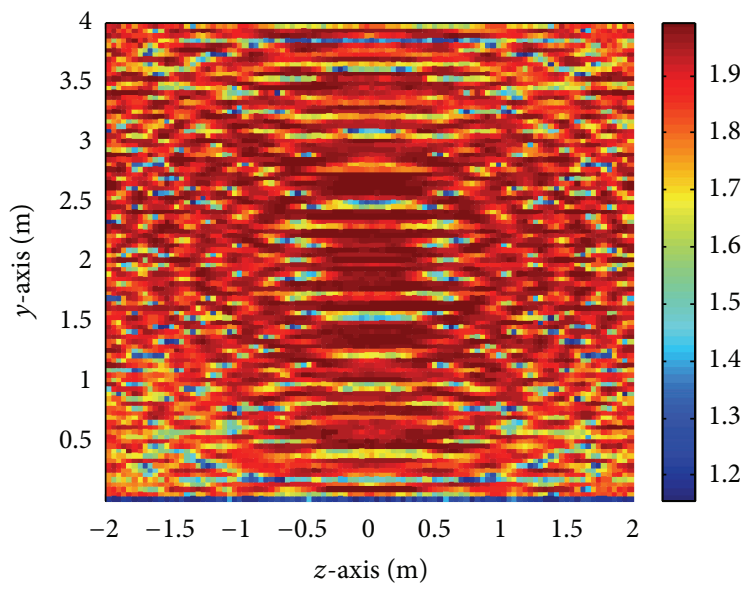

(c)

FIGURE 10: Capacity of the realistic DPD antenna on the cross section vertical to the $x$-axis in the TM channel. (a) $x=1 \lambda$. (b) $x=11 \lambda$. (c) $x=20 \lambda$.

maximum and the minimum singular values of the channel matrix $\mathbf{H}$. The CN characterizes invertibility of the channel matrix and shows how efficiently the multiple data streams can be decomposed [13]. A small value of the CN generally implies a well-conditioned channel matrix, which has approximately equal singular values. Thus, the value of $\mathrm{CN}$ gets closer to 1 , and the channel has a better performance of spatial multiplexing. Over the communication range within $2 \mathrm{~m}$, from Figure 17(a), the average values of $\mathrm{CN}$ in the $\mathrm{AC}$ and in the small room are 7.59 and 1.79, respectively, and, from Figure 17(b), the corresponding average values of $\mathrm{CN}$ are 4.04 and 7.31, respectively. We can see that, in the office room, with the same $\mathrm{Tx}$ and $\mathrm{Rx}$ antenna heights, the channel has the smallest values of $\mathrm{CN}$, which corresponds to the largest dual-polarized MIMO capacity. In Figure 18, the effect of the azimuth angel $\zeta$ between the Tx antenna and the $\mathrm{Rx}$ antenna is illustrated. During the experiment, the location of the Tx antenna is fixed, and the Rx antenna moves along a circle around the $\mathrm{Tx}$ antenna. The radius of the circle is firstly chosen as $10 \mathrm{~cm}$ and then as $20 \mathrm{~cm}$, and the corresponding results are compared. It is noted in Figure 18 that the dual-polarized MIMO capacities will fluctuate with $\zeta$, but the overall variations are similar for different communication range and different propagation scenario. The maximum capacity is about $30 \%$ larger than the minimum one.

\subsection{Channel Capacity Measurement Results of the Virtual} $D P L$. In order to study the effect of mutual coupling and the directions of loop polarization on the overall dual-polarized MIMO capacity, the experiments based on the virtual DPLs are also conducted. To be convenient for explanation, the loop is denoted by its axis; for example, the loop $x$ means the loop located on the $y z$ plane. The loops in the colocated structure mentioned above are corresponding to the case of loop $x$ and loop $z$.

Figures 19 and 20 show that, in the AC, the two orthogonal loops with ideal isolation can get a larger MIMO capacity than the two loops with mutual coupling in most cases. However, in the realistic office room, this is not the case. It is indicated that, in the $\mathrm{AC}$, the mutual coupling will decrease the dual-polarized MIMO capacity; however, in the 


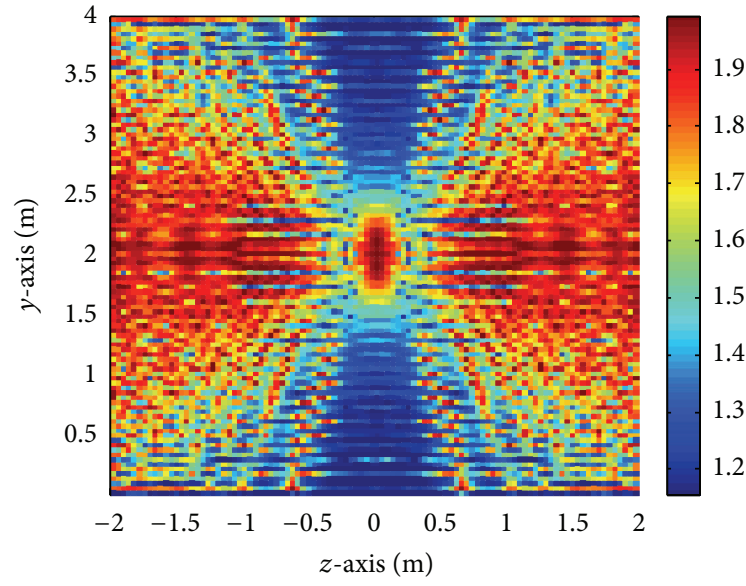

(a)

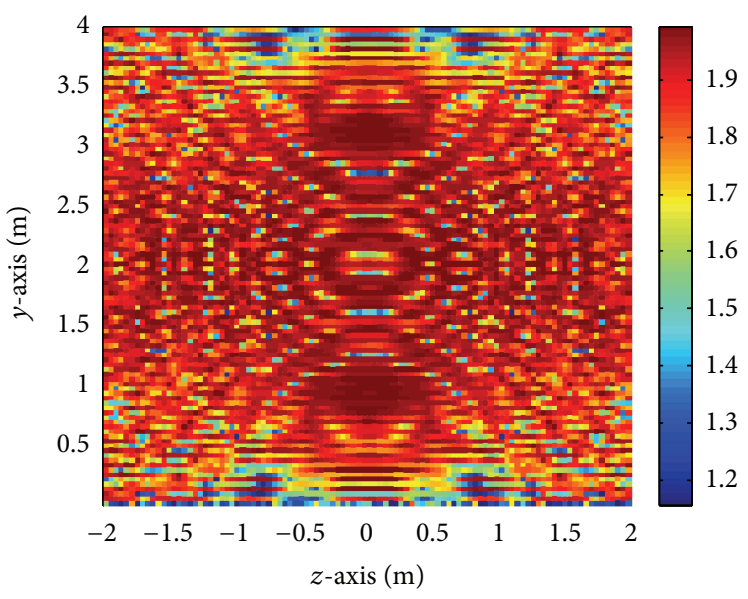

(b)

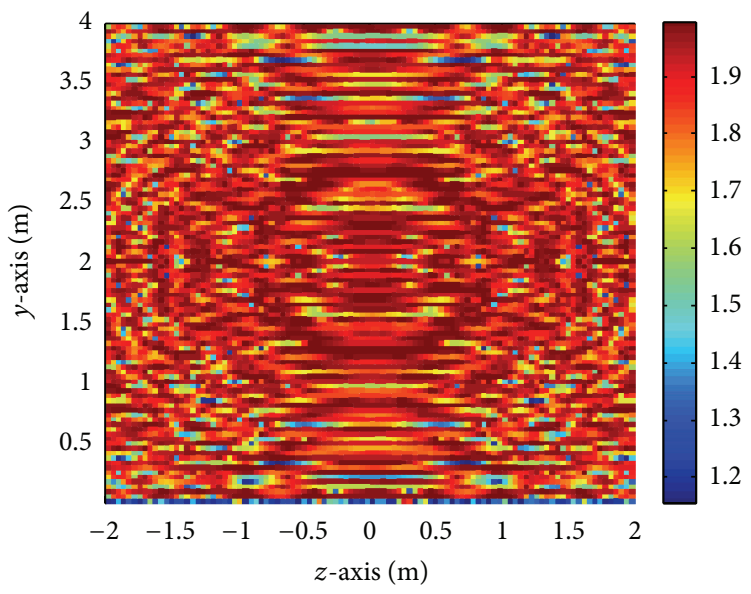

(c)

FIGURE 11: Capacity of the realistic DPL antenna on the cross section vertical to the $x$-axis in the TM channel. (a) $x=1 \lambda$. (b) $x=11 \lambda$. (c) $x=20 \lambda$.

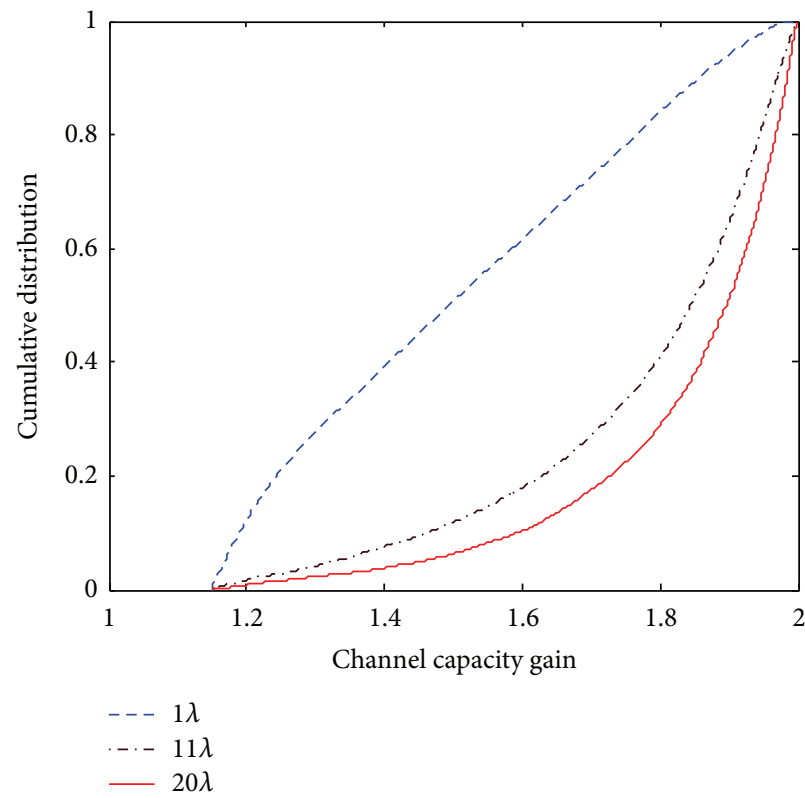

(a)

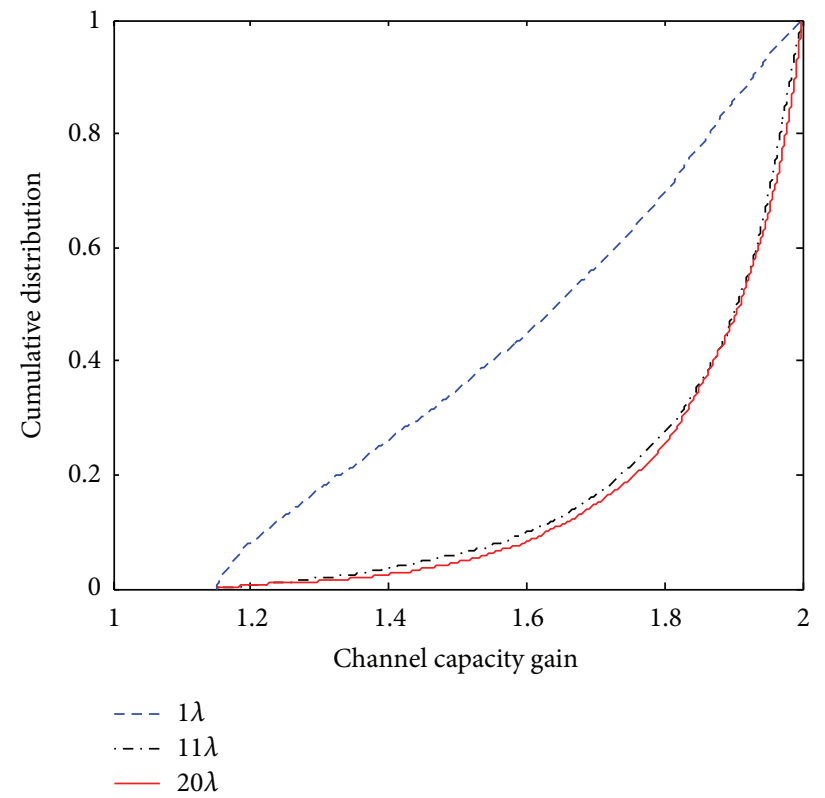

(b)

FIGURE 12: CDF of the capacity gain of the realistic antenna in the TM channel. (a) DPD. (b) DPL. 


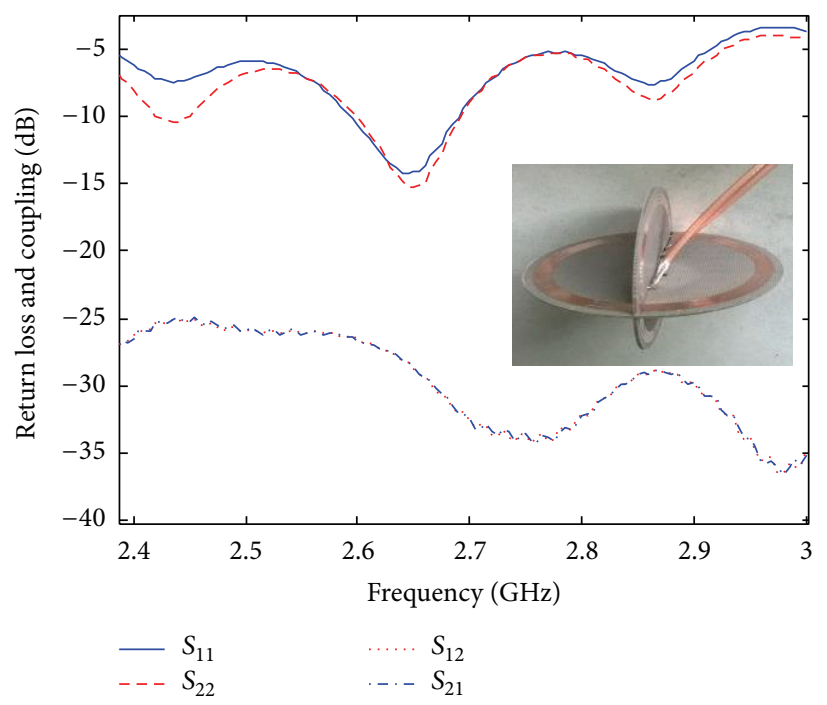

FIGURE 13: The measured scattering parameters of the DPL antenna.
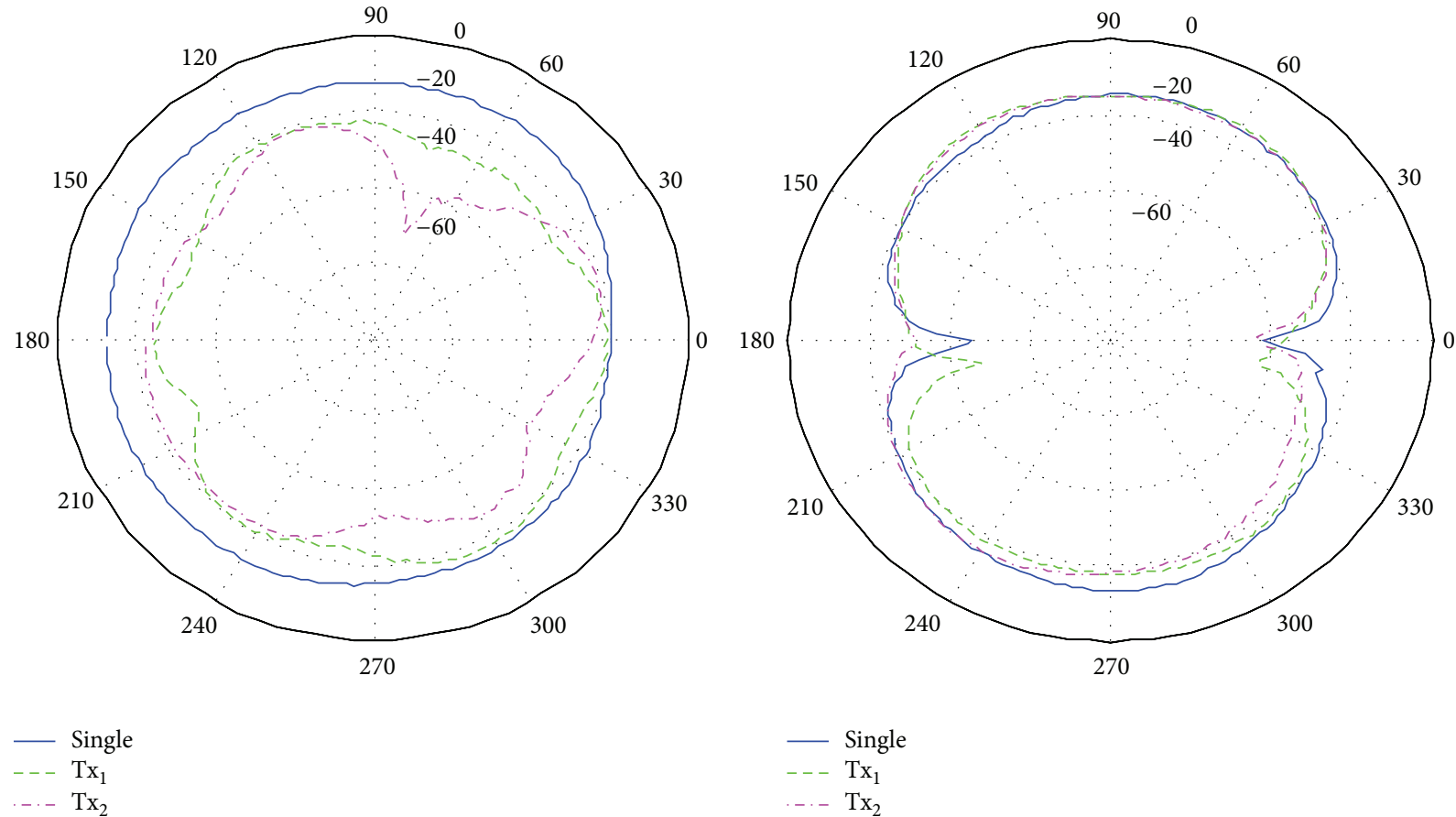

(a)

(b)

FIGURE 14: The measured radiation patterns of the DPL antenna. (a) E-plane. (b) H-plane.

real-world office room, the effect of mutual coupling is not always negative. Specifically, in Figure 20(a), the capacity of the colocated DPL is obviously larger than that of the virtual DPL with the same polarizations. Since it is in a multipath scenario, the overall multipolarized MIMO capacity is a complex interaction between the radiation pattern and the propagation properties of environment. More interestingly, Figures 19 and 20 also tell us that the selection of polarizations constituting the DPL also has a strong impact on the MIMO capacity, and the difference can reach $10-30 \%$, depending on the particular communication environment.

\section{Conclusion}

The DPD and DPL MIMO channel performances are evaluated by both the theoretical computations considering the ideal electric and magnetic dipoles and the numerical simulations considering the realistic DPD and DPL antenna. Computation results show that, for the ideal electric and magnetic dipoles, the dual-polarized MIMO systems have identical channel capacity. Specifically, capacity gain over the one-polarized antenna system shows that, in FS channel, the contour patterns of the capacity gain are regular concentric 


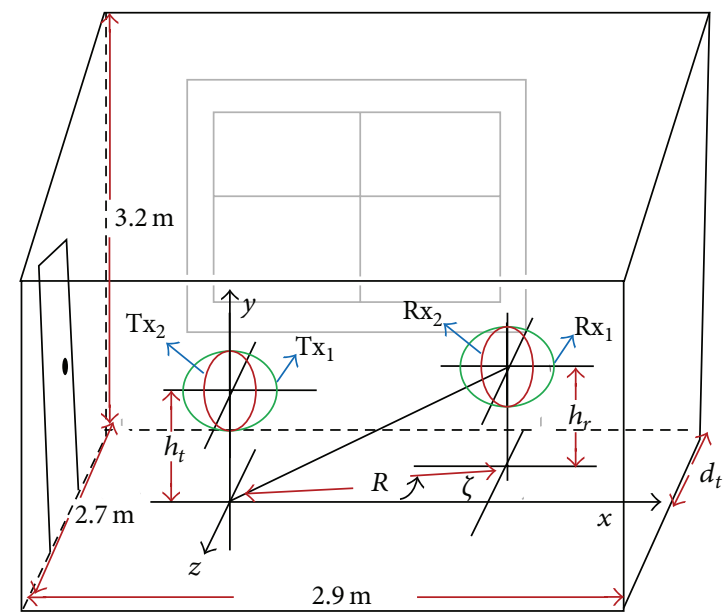

FIGURE 15: Measurement setup in the office room.

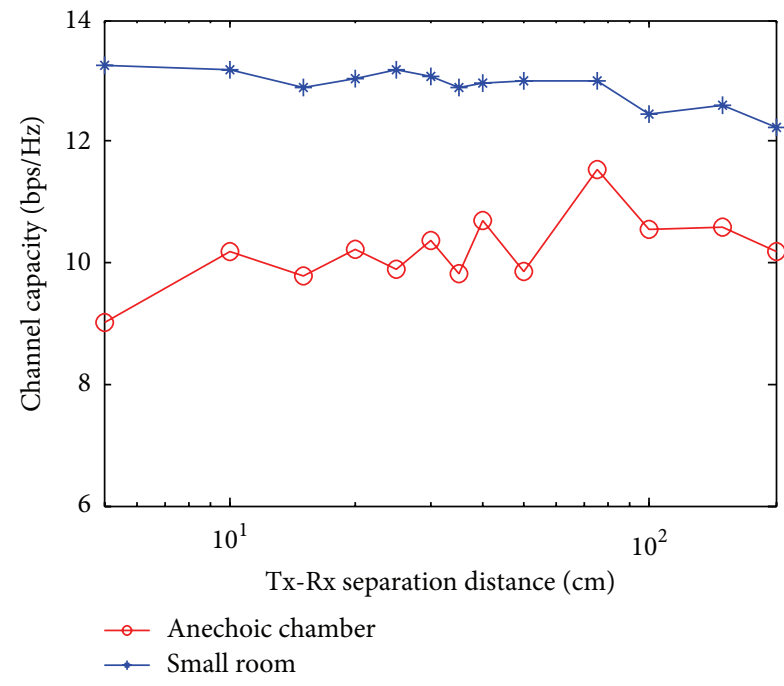

(a)

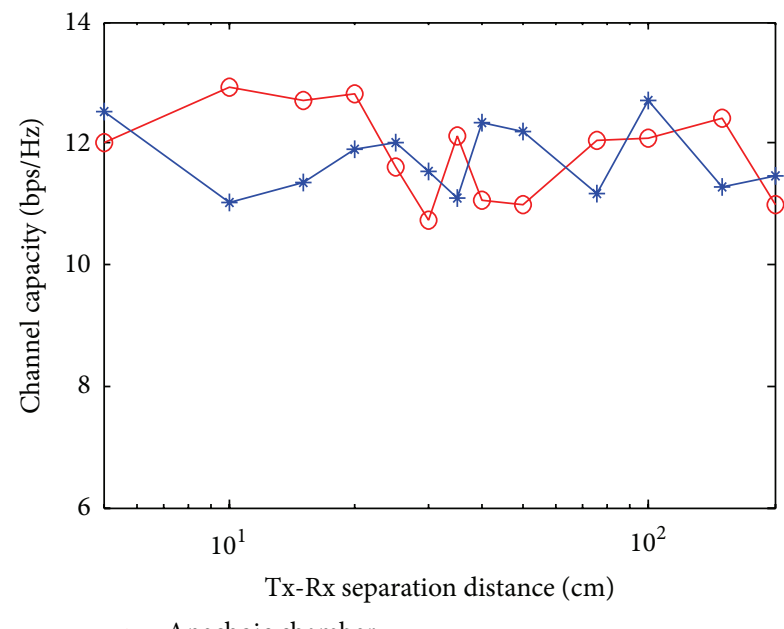

$\rightarrow$ Anechoic chamber

$\rightarrow$ Small room

(b)

FIgURE 16: Dependence of channel capacity on the communication distance. (a) $h_{t}=h_{r}=100 \mathrm{~cm}$. (b) $h_{t}=100 \mathrm{~cm}, h_{r}=70 \mathrm{~cm}$.

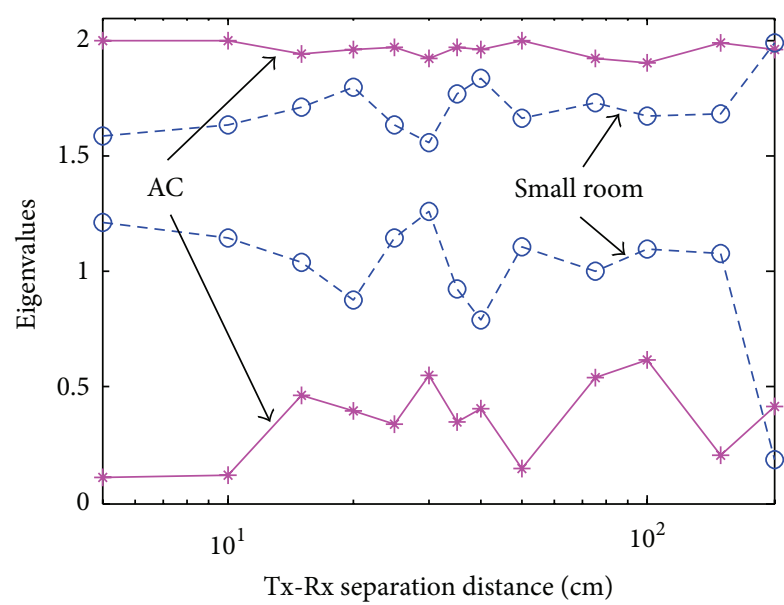

(a)

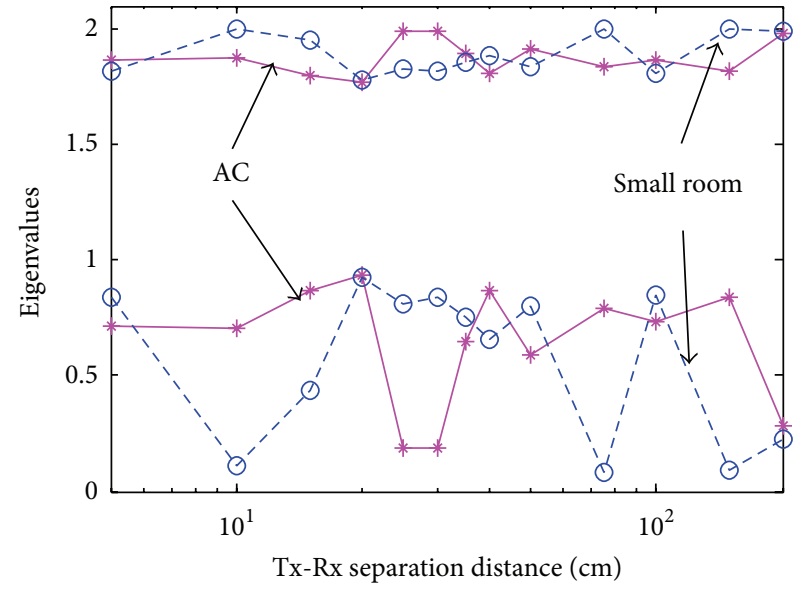

(b)

FIGURE 17: Dependence of the singular values of $\mathbf{H}$ on the communication distance. (a) $h_{t}=h_{r}=100 \mathrm{~cm}$. (b) $h_{t}=100 \mathrm{~cm}, h_{r}=70 \mathrm{~cm}$. 


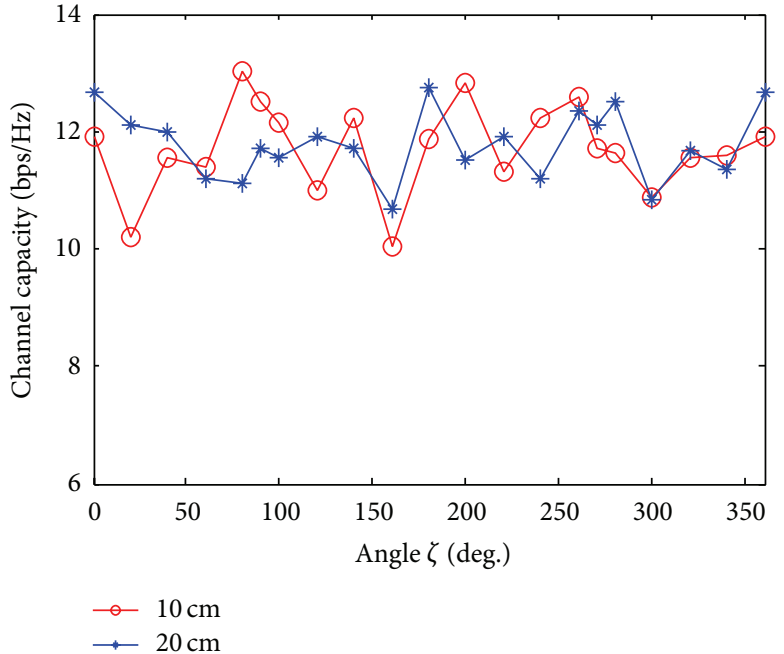

(a)

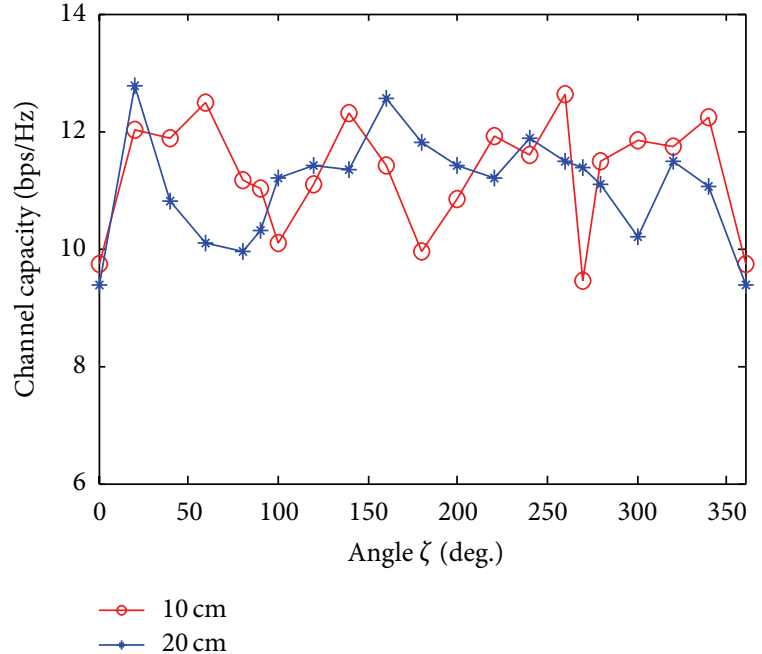

(b)

Figure 18: Dependence of channel capacity on the angle $\zeta$. (a) AC. (b) Small room.

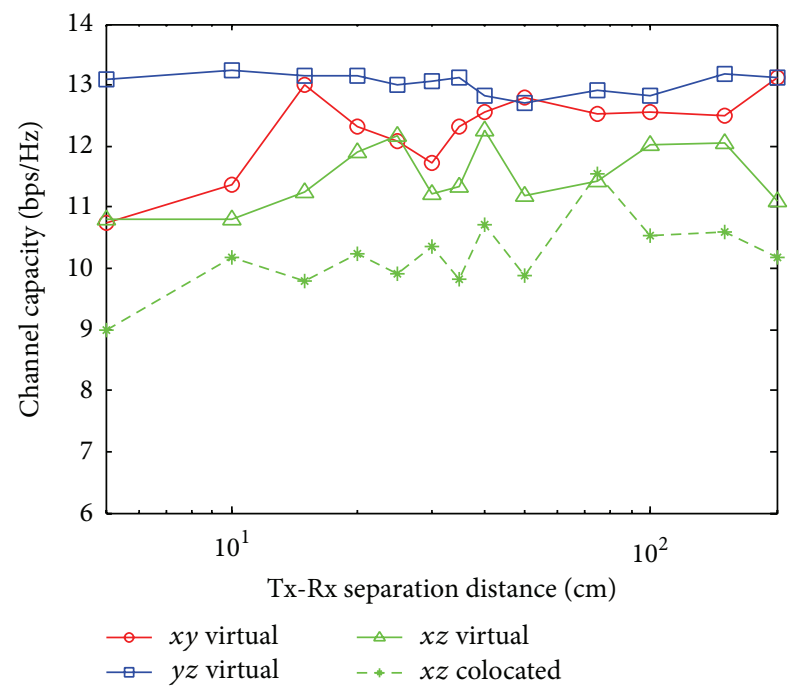

(a)

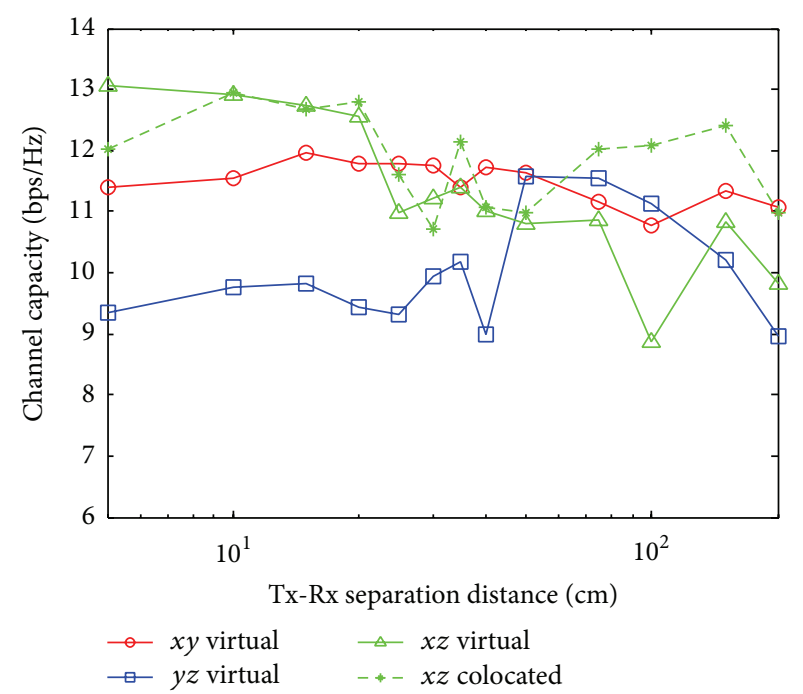

(b)

FIGURE 19: Channel capacity comparisons of the virtual and the colocated DPL antennas in the AC. (a) $h_{t}=h_{r}=100 \mathrm{~cm}$. (b) $h_{r}=100 \mathrm{~cm}$, $h_{r}=70 \mathrm{~cm}$.

circles, but, in the TM channel, they are not the regular circles and have a more complex pattern. But, for the realistic antennas, it is seen that the contour plots of the DPD and DPL antenna are different; however, both of them are similar to the results of the ideal antennas. Furthermore, the cumulative distribution function (CDF) of the capacity gain in the TM channel shows that, for small communication distance, the capacity gain obtained by the DPD antenna is obviously smaller than that of the DPL antenna, but, with the increase of the communication distance, the difference gets smaller.

A colocated DPL MIMO antenna with uniform current distribution along each loop is fabricated, which has a low mutual coupling. The capacity of this DPL is measured in both the AC and the real-world office room. The effects of communication distance and azimuthal angel between the $\mathrm{Tx}$ and $\mathrm{Rx}$ antennas are investigated. Computation results show that the capacity gains of using the DPL antenna with respect to the single-polarized antenna are 1.5-1.99, depending on the particular antenna location and the propagation properties of the scenario, which is basically consistent with the theoretical and the simulated results. The $\mathrm{CN}$ values are also computed, and it is shown that in the office room, with the same Tx and Rx antenna heights, the channel has the smallest values of $\mathrm{CN}$, which corresponds to the largest dual-polarized MIMO capacity. Furthermore, experiments based on the virtual DPLs are also conducted to study the effect of mutual 


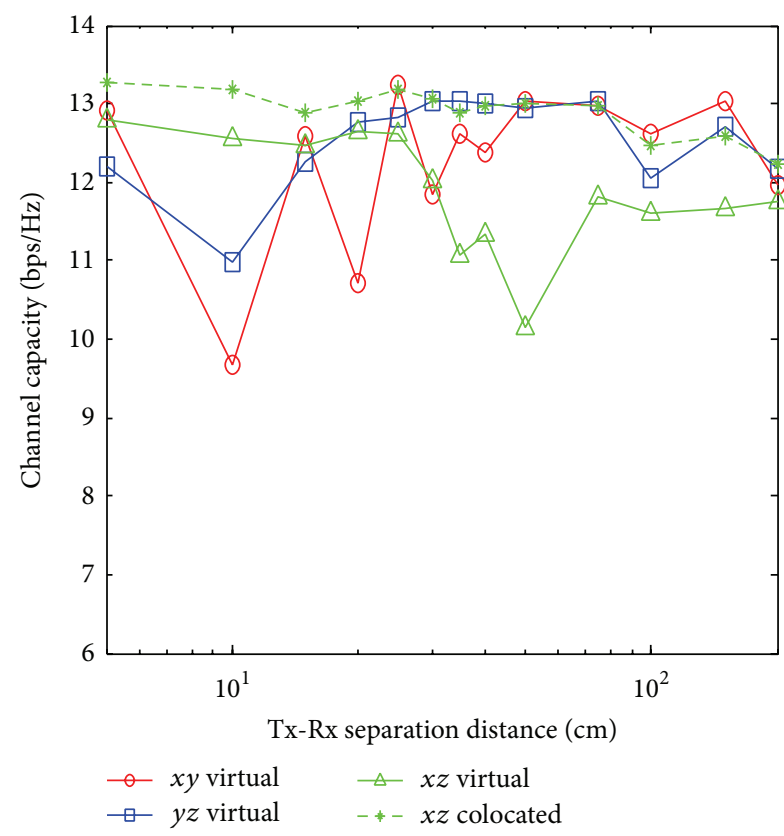

(a)

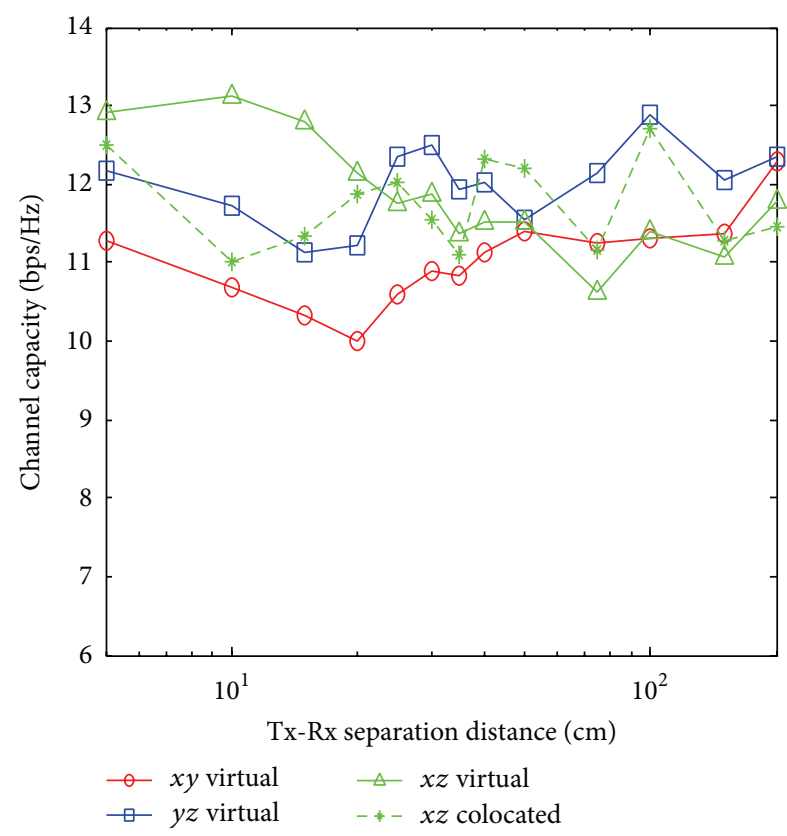

(b)

FIGURE 20: Channel capacity comparisons of the virtual and the colocated DPL antennas in the office room. (a) $h_{t}=h_{r}=100 \mathrm{~cm}$. (b) $h_{t}=100 \mathrm{~cm}, h_{r}=70 \mathrm{~cm}$.

coupling. Computation results show that, in the AC, the mutual coupling will generally decrease the dual-polarized MIMO capacity; however, in the realistic office room with rich multipath, the effect of mutual coupling is not always negative. More importantly, the selection of polarizations constituting the DPL also has a strong impact on the dualpolarized MIMO capacity.

\section{Conflict of Interests}

The authors declare that there is no conflict of interests regarding the publication of this paper.

\section{Acknowledgments}

This work was supported by the National Natural Science Foundations of China (Grant no. 61201235) and the Young Talents Program of Beijing Universities (no. YETP0595).

\section{References}

[1] M. R. Andrews, P. P. Mitra, and R. DeCarvalho, "Tripling the capacity of wireless communications using electromagnetic polarization," Nature, vol. 409, no. 6818, pp. 316-318, 2001.

[2] T. Svantesson, M. A. Jensen, and J. W. Wallace, "Analysis of electromagnetic field polarizations in multi-antenna systems," IEEE Transactions on Wireless Communications, vol. 3, no. 2, pp. 641-646, 2004.

[3] M. S. Elnaggar, S. K. Chaudhuri, and S. Safavi-Naeini, "Multipolarization dimensionality of multiantenna systems," Progress In Electromagnetics Research B, no. 14, pp. 45-63, 2009.
[4] A. S. Y. Poon and D. N. C. Tse, "Degree-of-freedom gain from using polarimetric antenna elements," IEEE Transactions on Information Theory, vol. 57, no. 9, pp. 5695-5709, 2011.

[5] D. Piao, "Characteristics of the hexapolarized MIMO channel over free-space and three non-free-space scenarios," IEEE Transactions on Wireless Communications, vol. 12, no. 8, pp. 4174-4182, 2013.

[6] A. S. Konanur, K. Gosalia, S. H. Krishnamurthy, B. Hughes, and G. Lazzi, "Increasing wireless channel capacity through MIMO systems employing co-located antennas," IEEE Transactions on Microwave Theory and Techniques, vol. 53, no. 6, pp. 1837-1844, 2005.

[7] G. Gupta, B. L. Hughes, and G. Lazzi, "On the degrees of freedom in linear array systems with tri-polarized antennas," IEEE Transactions on Wireless Communications, vol. 7, no. 7, pp. 2458-2462, 2008.

[8] B. Elnour and D. Erricolo, "A novel colocated cross-polarized two-loop PCB antenna in the ISM 2.4-GHz band," IEEE Antennas and Wireless Propagation Letters, vol. 9, pp. 1237-1240, 2010.

[9] D. Piao, Y. Mao, and H. Zhang, "Two novel colocated dualpolarized antennas with extremely low mutual coupling for polarization diversity MIMO applications," in Proceedings of the 2nd IEEE International Conference on Connected Vehicles and Expo (ICCVE '13), pp. 531-536, Las Vegas, Nev, USA, December 2013.

[10] D. Piao and L. Yang, "MIMO capacity comparisons of three types of colocated dual-polarized loop antennas," Progress in Electromagnetics Research C, vol. 54, pp. 41-48, 2014.

[11] K. Wei, Z. Zhang, and Z. Feng, "Design of a wideband horizontally polarized omnidirectional printed loop antenna," IEEE Antennas and Wireless Propagation Letters, vol. 11, pp. 49$52,2012$. 
[12] G. J. Foschini and M. J. Gans, "On limits of wireless communications in a fading environment when using multiple antennas," Wireless Personal Communications, vol. 6, no. 3, pp. 311-335, 1998.

[13] A. Kobyakov, M. Sauer, A. Ngoma, and J. H. Winters, "Effect of optical loss and antenna separation in $2 \times 2$ MIMO fiber-radio systems," IEEE Transactions on Antennas and Propagation, vol. 58, no. 1, pp. 187-194, 2010. 

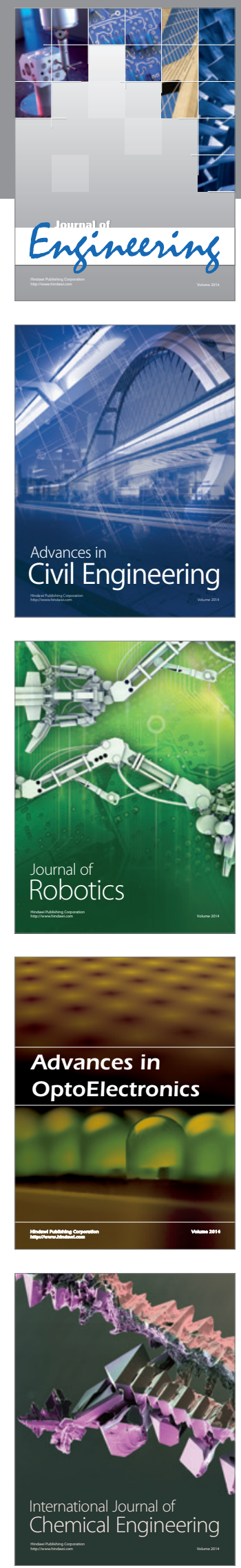

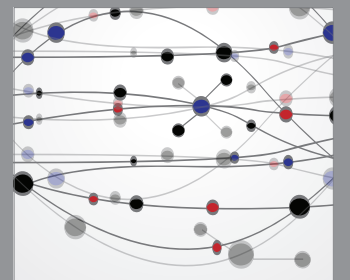

The Scientific World Journal
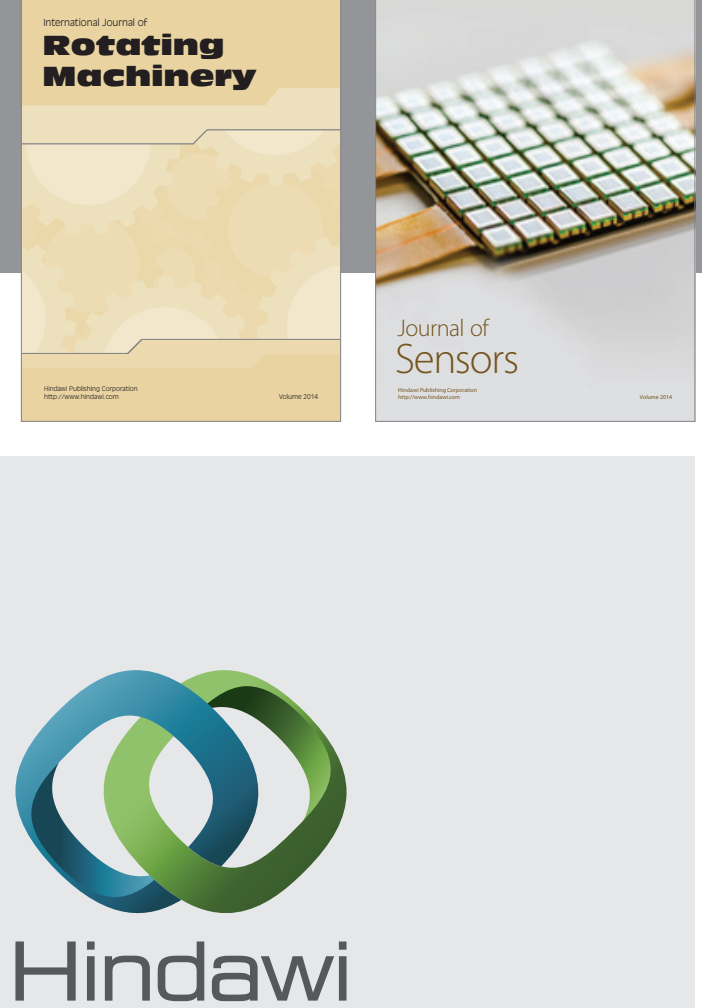

Submit your manuscripts at http://www.hindawi.com
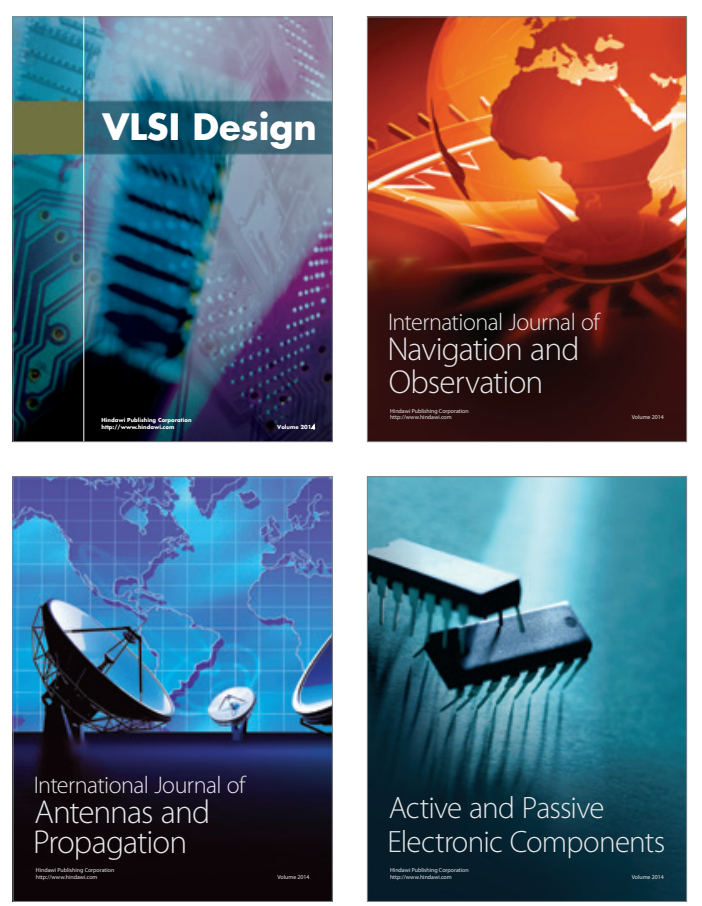
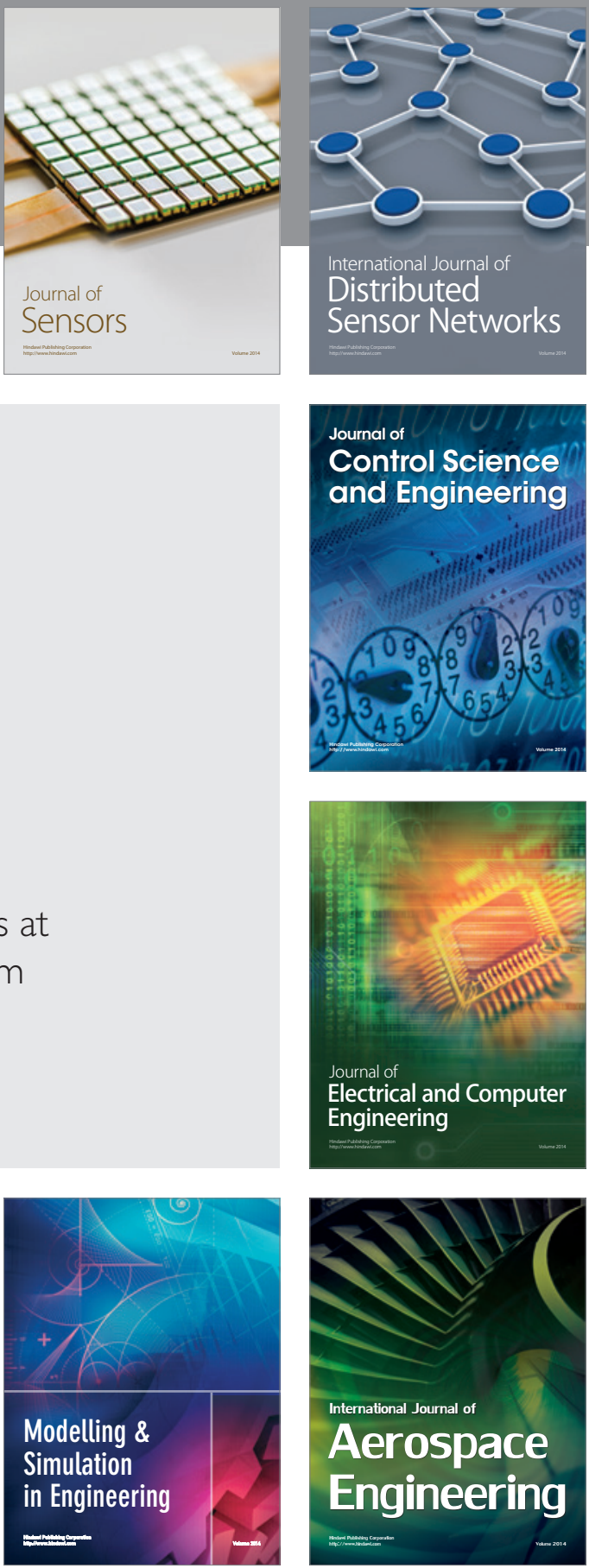

Journal of

Control Science

and Engineering
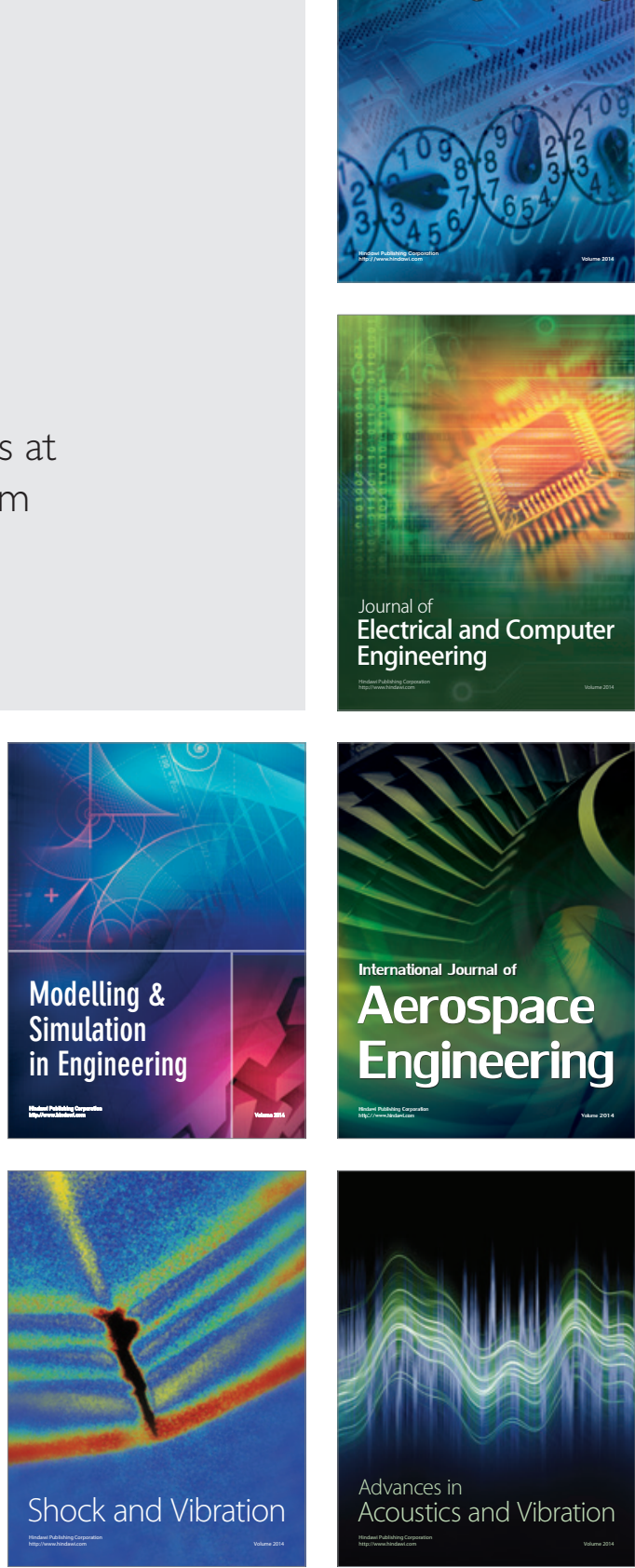